\title{
Local estimation of the conditional stable tail dependence
}

\section{function}

\author{
Mikael Escobar-Bach ${ }^{(1)}$, Yuri Goegebeur ${ }^{(1)}$, Armelle Guillou ${ }^{(2)}$ \\ (1) Department of Mathematics and Computer Science, University of Southern Denmark, Campusvej \\ 55, 5230 Odense M, Denmark \\ (2) Institut Recherche Mathématique Avancée, UMR 7501, Université de Strasbourg et CNRS, 7 rue \\ René Descartes, 67084 Strasbourg cedex, France
}

\begin{abstract}
We consider the local estimation of the stable tail dependence function when a random covariate is observed together with the variables of main interest. Our estimator is a weighted version of the empirical estimator adapted to the covariate framework. We provide the main asymptotic properties of our estimator, when properly normalized, in particular the convergence of the empirical process towards a tight centered Gaussian process. The finite sample performance of our estimator is illustrated on a small simulation study and on a dataset of air pollution measurements.
\end{abstract}

Keywords: Conditional stable tail dependence function, empirical process, stochastic convergence.

\section{Introduction}

A central topic in multivariate extreme value statistics is the estimation of the extremal dependence between two or more random variables. Ledford and Tawn (1997) introduced the coefficient of tail dependence as a summary measure of extremal dependence, and also proposed an estimator for this parameter. See also Peng (1999), Beirlant and Vandewalle (2002), Beirlant 
et al. (2011), Goegebeur and Guillou (2013), Dutang et al. (2014) for alternative estimators of this parameter. Other examples of summary dependence measures for extremes can be found in Coles et al. (1999). As an alternative to these summary measures, one can work with functions that give a complete characterisation of the extremal dependence, like the spectral distribution function (Einmahl et al., 1997), the Pickands dependence function (Pickands, 1981) or the stable tail dependence function (Huang, 1992). These functions can be seen as the analogues of copulas in classical statistics. In the present paper we focus on the stable tail dependence function.

For any arbitrary dimension $d$, let $\left(Y^{(1)}, \ldots, Y^{(d)}\right)$ be a multivariate random vector with continuous marginal distribution functions $F_{1}, \ldots, F_{d}$. The stable tail dependence function is defined for each $y_{i} \in \mathbb{R}_{+}, i=1, \ldots, d$, as

$$
\lim _{t \rightarrow \infty} t \mathbb{P}\left(1-F_{1}\left(Y^{(1)}\right) \leqslant t^{-1} y_{1} \quad \text { or } \quad \ldots \quad \text { or } \quad 1-F_{d}\left(Y^{(d)}\right) \leqslant t^{-1} y_{d}\right)=L\left(y_{1}, \ldots, y_{d}\right),
$$

provided that this limit exists, which can be rewritten as

$$
\lim _{t \rightarrow \infty} t\left[1-F\left(F_{1}^{-1}\left(1-t^{-1} y_{1}\right), \ldots, F_{d}^{-1}\left(1-t^{-1} y_{d}\right)\right)\right]=L\left(y_{1}, \ldots, y_{d}\right),
$$

where $F$ is the multivariate distribution function of the vector $\left(Y^{(1)}, \ldots, Y^{(d)}\right)$.

Now, consider a random sample of size $n$ drawn from $F$ and an intermediate sequence $k=k_{n}$, i.e. $k \rightarrow \infty$ as $n \rightarrow \infty$ with $k / n \rightarrow 0$. Let us denote $y=\left(y_{1}, \ldots, y_{d}\right)$ a vector of the positive quadrant $\mathbb{R}_{+}^{d}$ and $Y_{k, n}^{(j)}$ the $k$-th order statistic among $n$ realisations of the margins $Y^{(j)}, j=1, \ldots, d$. The empirical estimator of $L$ is then given by

$$
\left.\widehat{L}_{k}(y)=\frac{1}{k} \sum_{i=1}^{n} 1_{\left\{Y_{i}^{(1)} \geqslant Y_{n-\left[k y_{1}\right]+1, n}^{(1)}\right.} \quad \text { or } \quad \ldots \quad \text { or } \quad Y_{i}^{(d)} \geqslant Y_{n-\left[k y_{d}\right]+1, n}^{(d)}\right\} .
$$

The asymptotic behaviour of this estimator was first studied by Huang (1992); see also Drees and Huang (1998), de Haan and Ferreira (2006) and Bücher et al. (2014). We also refer to Peng (2010), Fougères et al. (2015), Beirlant et al. (2016) and Escobar-Bach et al. (2017b) where alternative estimators for $L$ were introduced. In the present paper we extend the empirical estimator to the situation where we observe a random covariate $X$ together with the variables of main interest $\left(Y^{(1)}, \ldots, Y^{(d)}\right)$. We consider thus a regression problem where we want to describe 
the extremal dependence between the random variables $\left(Y^{(1)}, \ldots, Y^{(d)}\right)$ given some observed value $x$ for the covariate $X$. Our approach is nonparametric and based on local estimation in the covariate space.

In the univariate context there is a quite extensive literature on estimation of tail parameters in presence of random covariates. In the framework of heavy-tailed distributions, nonparametric kernel methods were introduced by Daouia et al. (2011), who used a fixed number of extreme conditional quantile estimators to estimate the conditional extreme value index, for instance using the Hill (Hill, 1975) and Pickands (Pickands, 1975) estimators, whereas Goegebeur et al. (2014b) developed a nonparametric and asymptotically unbiased estimator based on weighted exceedances over a high threshold. The extension of this regression estimation of tail parameters to the full max-domain of attraction, has been considered in Daouia et al. (2013), who generalized Daouia et al. (2011), and also by Stupfler (2013) and Goegebeur et al. (2014a) where an adjustment of the moment estimator, originally proposed by Dekkers et al. (1989), to this setting of local estimation has been proposed. On the contrary, the development of extreme value methodology for regression problems with a multivariate response vector is still in its infancy. In de Carvalho and Davison (2014), a procedure was introduced to infer about extremal dependence in the presence of qualitative independent variables, that is, an ANOVAtype setting. Portier and Segers (2015) considered the estimation of a bivariate extreme value distribution under the simplifying assumption that the dependence between $Y^{(1)}$ and $Y^{(2)}$ does not depend on the value taken by the covariate, so that the dependence of the model on the covariates is only through the marginal distributions. Escobar-Bach et al. (2017a) studied the robust estimation of the conditional Pickands dependence function using the minimum density power divergence criterion, adapted to the context of local estimation. However, in that paper it is assumed that a random sample from a conditional bivariate extreme value distribution is available. In the present paper we relax this assumption and introduce a local estimator for the conditional stable tail dependence function assuming only that we have data available from a distribution with a dependence structure converging to that of an extreme value distribution. Thus, we extend the above framework to the case where the vector $\left(Y^{(1)}, \ldots, Y^{(d)}\right)$ is recorded 
along with a random covariate $X \in \mathbb{R}^{p}$. In that context, the stable tail dependence function together with the marginal distribution functions depend on the covariate $X$. In the sequel, for $j=1, \ldots, d$, we denote by $F_{j}(. \mid x)$, the continuous conditional distribution function of $Y^{(j)}$ given $X=x$ and $L(. \mid x)$ the conditional stable tail dependence function defined as

$\lim _{t \rightarrow \infty} t \mathbb{P}\left(1-F_{1}\left(Y^{(1)} \mid X\right) \leqslant t^{-1} y_{1}\right.$ or $\ldots$ or $\left.1-F_{d}\left(Y^{(d)} \mid X\right) \leqslant t^{-1} y_{d} \mid X=x\right)=L\left(y_{1}, \ldots, y_{d} \mid x\right)$

We establish the weak convergence of the empirical process of the properly normalized estimator using Donsker results for changing function classes and arguments based on the theory of Vapnik- $\breve{C}$ ervonenkis classes ( $V C$-classes). To the best of our knowledge this type of regression problem has not been considered in the multivariate extreme value literature.

The remainder of the paper is organised as follows. In the next section we introduce the local estimator for the conditional stable tail dependence function and study its asymptotic properties. In first instance we assume that the marginal conditional distribution functions are known, whereafter this assumption is removed and the unknown marginal conditional distribution functions are estimated locally using a kernel method. Finally, in Section 3, we illustrate the finite sample behaviour of our estimator with a small simulation study and on a dataset of air pollution measurements. All the proofs of the results are collected in the Appendix.

\section{Estimator and asymptotic properties}

Denote $(Y, X):=\left(Y^{(1)}, \ldots, Y^{(d)}, X\right)$, a random vector satisfying $(1)$, and let $\left(Y_{1}, X_{1}\right), \ldots,\left(Y_{n}, X_{n}\right)$, be independent copies of $(Y, X)$, where $X \in \mathbb{R}^{p}$ has density function $f$. As is usual in the extreme value context, we consider an intermediate sequence $k=k_{n}$, i.e. $k \rightarrow \infty$ as $n \rightarrow \infty$ with $k / n \rightarrow 0$. Let us denote $y:=\left(y_{1}, \ldots, y_{d}\right)$ a vector of the positive quadrant $\mathbb{R}_{+}^{d}$. The event $A_{t, y}$ is defined for any $t \geqslant 0$ and $y \in \mathbb{R}_{+}^{d}$ as

$$
A_{t, y}:=\left\{1-F_{1}\left(Y^{(1)} \mid X\right) \leqslant t^{-1} y_{1} \text { or } \ldots \text { or } 1-F_{d}\left(Y^{(d)} \mid X\right) \leqslant t^{-1} y_{d}\right\}
$$


and $A_{t, y}^{(i)}$ denotes its analogue for observation $\left(Y_{i}, X_{i}\right), i=1, \ldots, n$. The conditional empirical estimator is then given for any $x \in \mathbb{R}^{p}$ by

$$
\begin{aligned}
\widehat{T}_{k}(y \mid x) & :=\frac{1}{k} \sum_{i=1}^{n} K_{h}\left(x-X_{i}\right) \mathbb{1}_{\left\{1-F_{1}\left(Y_{i}^{(1)} \mid X_{i}\right) \leqslant \frac{k}{n} y_{1} \text { or } \ldots \text { or } 1-F_{d}\left(Y_{i}^{(d)} \mid X_{i}\right) \leqslant \frac{k}{n} y_{d}\right\}} \\
& =\frac{1}{k} \sum_{i=1}^{n} K_{h}\left(x-X_{i}\right) \mathbb{1}_{A_{n / k, y}^{(i)}},
\end{aligned}
$$

where $K_{h}():.=K(. / h) / h^{p}$ with $K$ a joint density function and $h=h_{n}$ is a positive non-random sequence satisfying $h_{n} \rightarrow 0$ as $n \rightarrow \infty$.

The aim of the paper is to derive stochastic convergence results for empirical processes based on (2), with $y \in[0, T]^{d}, T>0$, but with the covariate argument fixed, meaning that we will focus our study only around one reference position $x_{0} \in \operatorname{Int}\left(S_{X}\right)$, the interior of the support $S_{X}$ of $f$. In order to derive the asymptotic behaviour of $\widehat{T}_{k}\left(y \mid x_{0}\right)$, we need to introduce some conditions mentioned below and well-known in the extreme value framework. Let $\|\cdot\|$ be some norm on $\mathbb{R}^{p}$, and denote by $B_{x}(r)$ the closed ball with respect to $\|$.$\| centered at x$ and radius $r>0$.

First order condition: The limit in (1) exists for all $x \in S_{X}$ and $y \in \mathbb{R}_{+}^{d}$, and the convergence is uniform on $[0, T]^{d} \times B_{x_{0}}(r)$ for any $T>0$ and $a r>0$.

Second order condition: For any $x \in S_{X}$ there exist a positive function $\alpha_{x}$ such that $\alpha_{x}(t) \rightarrow 0$ as $t \rightarrow \infty$ and a non null function $M_{x}$ such that for all $y \in \mathbb{R}_{+}^{d}$

$$
\lim _{t \rightarrow \infty} \frac{1}{\alpha_{x}(t)}\left\{t \mathbb{P}\left(A_{t, y} \mid X=x\right)-L(y \mid x)\right\}=M_{x}(y),
$$

uniformly on $[0, T]^{d} \times B_{x_{0}}(r)$ for any $T>0$ and a $r>0$.

Due to the regression context, we need some Hölder-type conditions.

Assumption (D). There exist $M_{f}>0$ and $\eta_{f}>0$ such that $|f(x)-f(z)| \leqslant M_{f}\|x-z\| \eta_{f}$, for all $(x, z) \in S_{X} \times S_{X}$.

Assumption $(\mathcal{L})$. There exist $M_{L}>0$ and $\eta_{L}>0$ such that $|L(y \mid x)-L(y \mid z)| \leqslant M_{L}\|x-z\|^{\eta_{L}}$, for all $(x, z) \in B_{x_{0}}(r) \times B_{x_{0}}(r), r>0$, and $y \in[0, T]^{d}, T>0$. 
Assumption $(\mathcal{A})$. There exist $M_{\alpha}>0$ and $\eta_{\alpha}>0$ such that $\left|\alpha_{x}(t)-\alpha_{z}(t)\right| \leqslant M_{\alpha}\|x-z\|^{\eta_{\alpha}}$, for all $(x, z) \in S_{X} \times S_{X}$ and $t \geqslant 0$.

Also a usual condition is assumed on the kernel function $K$.

Assumption $\left(\mathcal{K}_{1}\right) . K$ is a bounded density function on $\mathbb{R}^{p}$ with support $S_{K}$ included in the unit ball of $\mathbb{R}^{p}$ with respect to the norm $\|$.$\| .$

\subsection{Marginal conditional distributions known}

In this section, we restrict our interest to the case where the marginal conditional distribution functions $F_{j}(. \mid x), j=1, \ldots, d$, are known. We start by showing the convergence in probability of our main statistic under some weak assumptions.

Lemma 2.1 Let $y \in \mathbb{R}_{+}^{d}$. Assume the first order condition, $\left(\mathcal{K}_{1}\right)$ and that the functions $f$ and $x \rightarrow L(y \mid x)$ are continuous at $x_{0} \in \operatorname{Int}\left(S_{X}\right)$ non-empty. If for $n \rightarrow \infty$ we have $k \rightarrow \infty$ and $h \rightarrow 0$ in such a way that $k / n \rightarrow 0$ and $k h^{p} \rightarrow \infty$, then for $x_{0}$ such that $f\left(x_{0}\right)>0$, we have

$$
\widehat{T}_{k}\left(y \mid x_{0}\right) \stackrel{\mathbb{P}}{\longrightarrow} f\left(x_{0}\right) L\left(y \mid x_{0}\right) .
$$

This result indicates that in order to estimate $L\left(y \mid x_{0}\right)$, the statistic $\widehat{T}_{k}\left(y \mid x_{0}\right)$ will need to be divided by an estimator for $f\left(x_{0}\right)$. Our main objective in this section is to show the weak convergence of the stochastic process

$$
\left\{\sqrt{k h^{p}}\left(\frac{\widehat{T}_{k}\left(y \mid x_{0}\right)}{\widehat{f}_{n}\left(x_{0}\right)}-L\left(y \mid x_{0}\right)-\alpha_{x_{0}}\left(\frac{n}{k}\right) M_{x_{0}}(y)\right), y \in[0, T]^{d}\right\},
$$

for any $T>0$, where $\widehat{f}_{n}$ is the usual kernel density function estimator for $f$ :

$$
\widehat{f}_{n}(x):=\frac{1}{n} \sum_{i=1}^{n} K_{h}\left(x-X_{i}\right)
$$

see e.g. Parzen (1962). Note that for convenience we use here for $\widehat{f}_{n}$ the same kernel function $K$ and bandwidth parameter $h$ as for $\widehat{T}_{k}\left(y \mid x_{0}\right)$.

As a preliminary step we deduce the covariance structure of the limiting process (apart from the scaling by $\left.1 / \widehat{f}_{n}\left(x_{0}\right)\right)$. 
Lemma 2.2 Under the assumptions of Lemma 2.1, we have for any $y, y^{\prime} \in \mathbb{R}_{+}^{d}$

$k h^{p} \operatorname{Cov}\left(\widehat{T}_{k}\left(y \mid x_{0}\right), \widehat{T}_{k}\left(y^{\prime} \mid x_{0}\right)\right) \rightarrow f\left(x_{0}\right)\|K\|_{2}^{2}\left(L\left(y \mid x_{0}\right)+L\left(y^{\prime} \mid x_{0}\right)-L\left(y \vee y^{\prime} \mid x_{0}\right)\right)$, as $n \rightarrow \infty$.

Here, $y \vee y^{\prime}:=\left(y_{1} \vee y_{1}^{\prime}, y_{2} \vee y_{2}^{\prime}, \ldots, y_{d} \vee y_{d}^{\prime}\right)$ and $\|K\|_{2}:=\sqrt{\int_{S_{K}} K^{2}(u) d u}$.

We derive now the weak convergence of (3) using Donsker type results for empirical processes with changing function classes and arguments based on the theory of $V C$-classes, as formulated in van der Vaart and Wellner (1996). These allow us to obtain weak convergence results by mainly focusing on the class of functions involved in our estimator. It should be mentioned that our main weak convergence results are derived in the usual Skorohod space, here $\mathcal{D}\left([0, T]^{d}\right)$ equipped with the sup norm $\|\cdot\|_{\infty}$.

Theorem 2.1 Assume the second order condition, $(\mathcal{D}),(\mathcal{L}),(\mathcal{A}),\left(\mathcal{K}_{1}\right)$, and $(x, y) \rightarrow M_{x}(y)$ being continuous on $B_{x_{0}}(r) \times[0, T]^{d}$, with $B_{x_{0}}(r) \subset S_{X}$. Consider sequences $k \rightarrow \infty$ and $h \rightarrow 0$ as $n \rightarrow \infty$, in such a way that $k / n \rightarrow 0, k h^{p} \rightarrow \infty$ and

$$
\sqrt{k h^{p}} h^{\min \left(\eta_{f}, \eta_{L}, \eta_{\alpha}\right)} \rightarrow 0 \text { and } \sqrt{k h^{p}} \alpha_{x_{0}}(n / k) \rightarrow \lambda_{x_{0}} \in \mathbb{R}_{+} .
$$

Then, for $x_{0}$ such that $f\left(x_{0}\right)>0$, the process

$$
\left\{\sqrt{k h^{p}}\left(\frac{\widehat{T}_{k}\left(y \mid x_{0}\right)}{\widehat{f}_{n}\left(x_{0}\right)}-L\left(y \mid x_{0}\right)-\alpha_{x_{0}}\left(\frac{n}{k}\right) M_{x_{0}}(y)\right), y \in[0, T]^{d}\right\},
$$

weakly converges in $\mathcal{D}\left([0, T]^{d}\right)$ towards a tight centered Gaussian process $\left\{B_{y}, y \in[0, T]^{d}\right\}$, for any $T>0$, with covariance structure given by

$$
\operatorname{Cov}\left(B_{y}, B_{y^{\prime}}\right)=\frac{\|K\|_{2}^{2}}{f\left(x_{0}\right)}\left(L\left(y \mid x_{0}\right)+L\left(y^{\prime} \mid x_{0}\right)-L\left(y \vee y^{\prime} \mid x_{0}\right)\right)
$$

where $y, y^{\prime} \in[0, T]^{d}$.

\subsection{Marginal conditional distributions unknown}

In this section, we consider the general framework where all $F_{j}(. \mid x), j=1, \ldots, d$, are unknown conditional distribution functions. We want to mimic what has been done in the previous section 
in case where these conditional distributions are assumed to be known. To this aim, we consider the random vectors

$$
\left(\widehat{F}_{n, 1}\left(Y_{i}^{(1)} \mid X_{i}\right), \widehat{F}_{n, 2}\left(Y_{i}^{(2)} \mid X_{i}\right), \ldots, \widehat{F}_{n, d}\left(Y_{i}^{(d)} \mid X_{i}\right), X_{i}\right), \quad i=1, \ldots, n,
$$

for suitable estimators $\widehat{F}_{n, j}$ of $F_{j}, j=1, \ldots, d$. Then, similarly as in Section 2.1, we study the statistic

$$
\breve{T}_{k}\left(y \mid x_{0}\right):=\frac{1}{k} \sum_{i=1}^{n} K_{h}\left(x_{0}-X_{i}\right) \mathbb{1}_{\left\{1-\widehat{F}_{n, 1}\left(Y_{i}^{(1)} \mid X_{i}\right) \leqslant \frac{k}{n} y_{1} \text { or } \ldots \text { or } 1-\widehat{F}_{n, d}\left(Y_{i}^{(d)} \mid X_{i}\right) \leqslant \frac{k}{n} y_{d}\right\}} .
$$

Our final goal is still the same, that is the weak convergence of the stochastic process

$$
\left\{\sqrt{k h^{p}}\left(\frac{\breve{T}_{k}\left(y \mid x_{0}\right)}{\widehat{f}_{n}\left(x_{0}\right)}-L\left(y \mid x_{0}\right)-\alpha_{x_{0}}\left(\frac{n}{k}\right) M_{x_{0}}(y)\right), y \in[0, T]^{d}\right\} .
$$

The idea will be to decompose the process $\left\{\sqrt{k h^{p}}\left(\breve{T}_{k}-\mathbb{E}\left[\breve{T}_{k}\right]\right)\left(y \mid x_{0}\right), y \in[0, T]^{d}\right\}$ into the two terms

$$
\left\{\sqrt{k h^{p}}\left(\widehat{T}_{k}-\mathbb{E}\left[\widehat{T}_{k}\right]\right)\left(y \mid x_{0}\right)+\sqrt{k h^{p}}\left(\left[\breve{T}_{k}-\widehat{T}_{k}\right]-\mathbb{E}\left[\breve{T}_{k}-\widehat{T}_{k}\right]\right)\left(y \mid x_{0}\right), y \in[0, T]^{d}\right\} .
$$

The first term in the above display can be dealt with using the results of Section 2.1 whereas we have to show that the second term is uniformly negligible. To achieve this objective, let us introduce the following empirical kernel estimator of the unknown conditional distribution functions

$$
\widehat{F}_{n, j}(y \mid x):=\frac{\sum_{i=1}^{n} K_{c}\left(x-X_{i}\right) \mathbb{1}_{\left\{Y_{i}^{(j)} \leqslant y\right\}}}{\sum_{i=1}^{n} K_{c}\left(x-X_{i}\right)}, j=1, \ldots, d,
$$

where $c:=c_{n}$ is a positive non-random sequence satisfying $c_{n} \rightarrow 0$ as $n \rightarrow \infty$. Here we kept the same kernel $K$ as for $\breve{T}_{k}\left(y \mid x_{0}\right)$, but of course any other kernel function can be used.

We need to impose again some assumptions, in particular a Hölder-type condition on each marginal conditional distribution function $F_{j}$ similar to the one imposed on the conditional stable tail dependence function.

Assumption $\left(\mathcal{F}_{m}\right)$. There exist $M_{F_{j}}>0$ and $\eta_{F_{j}}>0$ such that $\left|F_{j}(y \mid x)-F_{j}(y \mid z)\right| \leqslant$ $M_{F_{j}}\|x-z\|^{\eta_{F_{j}}}$, for all $y \in \mathbb{R}$, all $(x, z) \in S_{X} \times S_{X}$ and $j=1, \ldots, d$. 
Concerning the kernel $K$ a stronger assumption than $\left(\mathcal{K}_{1}\right)$ is needed.

Assumption $\left(\mathcal{K}_{2}\right) . K$ satisfies Assumption $\left(\mathcal{K}_{1}\right)$, there exists $\delta, m>0$ such that $B_{0}(\delta) \subset S_{K}$ and $K(u) \geqslant m$ for all $u \in B_{0}(\delta)$, and $K$ belongs to the linear span (the set of finite linear combinations) of functions $k \geqslant 0$ satisfying the following property: the subgraph of $k,\{(s, u)$ : $k(s) \geqslant u\}$, can be represented as a finite number of Boolean operations among sets of the form $\{(s, u): q(s, u) \geqslant \varphi(u)\}$, where $q$ is a polynomial on $\mathbb{R}^{p} \times \mathbb{R}$ and $\varphi$ is an arbitrary real function.

This assumption has already been used in Giné and Guillou (2002) and Escobar-Bach et al. (2017a). In particular, we refer to the latter to enunciate the following lemma that measures the discrepancy between the conditional distribution function $F_{j}$ and its empirical kernel version $\widehat{F}_{n, j}$.

Lemma 2.3 Assume that there exists $b>0$ such that $f(x) \geqslant b, \forall x \in S_{X} \subset \mathbb{R}^{p}, f$ is bounded, and $\left(\mathcal{K}_{2}\right)$ and $\left(\mathcal{F}_{m}\right)$ hold. Consider a sequence $c$ tending to 0 as $n \rightarrow \infty$ such that for some $q>1$

$$
\frac{|\log c|^{q}}{n c^{p}} \longrightarrow 0 .
$$

Also assume that there exists an $\varepsilon>0$ such that for $n$ sufficiently large

$$
\inf _{x \in S_{X}} \lambda\left(\left\{u \in B_{0}(1): x-c u \in S_{X}\right\}\right)>\varepsilon,
$$

where $\lambda$ denotes the Lebesgue measure. Then for any $0<\eta<\min \left(\eta_{F_{1}}, \ldots, \eta_{F_{d}}\right)$, we have

$$
\sup _{(y, x) \in \mathbb{R} \times S_{X}}\left|\widehat{F}_{n, j}(y \mid x)-F_{j}(y \mid x)\right|=o_{\mathbb{P}}\left(\max \left(\sqrt{\frac{\mid \log c^{q}}{n c^{p}}}, c^{\eta}\right)\right), \text { for } j=1, \ldots, d \text {. }
$$

This rate of convergence allows us to study the second term in (4) and to show that it is uniformly negligible.

Theorem 2.2 Assume that there exists $b>0$ such that $f(x) \geqslant b, \forall x \in S_{X} \subset \mathbb{R}^{p}, f$ is bounded, $\left(\mathcal{K}_{2}\right),\left(\mathcal{F}_{m}\right),(\mathcal{D})$, the first order condition and condition (5), and also for any $y \in[0, T]^{d}$ that $x \rightarrow L(y \mid x)$ continuous at $x_{0} \in \operatorname{Int}\left(S_{X}\right)$ non-empty. Consider sequences $k \rightarrow \infty, h \rightarrow 0$ and $c \rightarrow 0$ as $n \rightarrow \infty$, such that $k / n \rightarrow 0, k h^{p} \rightarrow \infty$, and with for some $q>1$ and $0<\eta<$ $\min \left(\eta_{F_{1}}, \ldots, \eta_{F_{d}}\right)$

$$
n \sqrt{\frac{h^{p}}{k}} r_{n}:=n \sqrt{\frac{h^{p}}{k}} \max \left(\sqrt{\frac{\mid \log c^{q}}{n c^{p}}}, c^{\eta}\right) \longrightarrow 0 \text {, as } n \rightarrow \infty .
$$


Then

$$
\sup _{y \in[0, T]^{d}} \sqrt{k h^{p}}\left|\breve{T}_{k}-\widehat{T}_{k}-\mathbb{E}\left[\breve{T}_{k}-\widehat{T}_{k}\right]\right|\left(y \mid x_{0}\right)=o_{\mathbb{P}}(1)
$$

Finally, the decomposition (4) combined with Theorem 2.2 and the results from Section 2.1, yield the desired final result of this paper.

Theorem 2.3 Assume the second order condition, $(x, y) \rightarrow M_{x}(y)$ continuous on $B_{x_{0}}(r) \times$ $[0, T]^{d}$, with $B_{x_{0}}(r) \subset S_{X}$, and that there exists $b>0$ with $f(x) \geqslant b, \forall x \in S_{X} \subset \mathbb{R}^{p}, f$ bounded. Under $(\mathcal{D}),(\mathcal{L}),(\mathcal{A}),\left(\mathcal{F}_{m}\right),\left(\mathcal{K}_{2}\right)$ and condition $(5)$, consider sequences $k \rightarrow \infty, h \rightarrow 0$ and $c \rightarrow 0$ as $n \rightarrow \infty$, such that $k / n \rightarrow 0, k h^{p} \rightarrow \infty$ with

$$
\sqrt{k h^{p}} h^{\min \left(\eta_{f}, \eta_{L}, \eta_{\alpha}\right)} \rightarrow 0, \quad \sqrt{k h^{p}} \alpha_{x_{0}}(n / k) \rightarrow \lambda_{x_{0}} \in \mathbb{R}_{+}
$$

and for some $q>1$ and $0<\eta<\min \left(\eta_{F_{1}}, \ldots, \eta_{F_{d}}\right)$

$$
n \sqrt{\frac{h^{p}}{k}} \max \left(\sqrt{\frac{|\log c|^{q}}{n c^{p}}}, c^{\eta}\right) \longrightarrow 0 .
$$

Then, the process

$$
\left\{\sqrt{k h^{p}}\left(\frac{\breve{T}_{k}\left(y \mid x_{0}\right)}{\widehat{f}_{n}\left(x_{0}\right)}-L\left(y \mid x_{0}\right)-\alpha_{x_{0}}\left(\frac{n}{k}\right) M_{x_{0}}(y)\right), y \in[0, T]^{d}\right\},
$$

weakly converges in $\mathcal{D}\left([0, T]^{d}\right)$ towards a tight centered Gaussian process $\left\{B_{y}, y \in[0, T]^{d}\right\}$, for any $T>0$, with covariance structure given in Theorem 2.1.

\section{Simulation and real data analysis}

\subsection{A small simulation study}

Our aim in this section is to illustrate the finite sample behaviour of our estimator

$$
\bar{L}_{k}(y \mid x):=\frac{\breve{T}_{k}(y \mid x)}{\widehat{f}_{n}(x)}
$$

with a small simulation study. We focus on dimension $d=2$ and we consider the two following models: 
- Model 1: We consider the bivariate Student distribution with density function

$$
f_{Y_{1}, Y_{2}}\left(y_{1}, y_{2}\right)=\frac{\sqrt{1-\theta^{2}}}{2 \pi}\left(1+\frac{y_{1}^{2}-2 \theta y_{1} y_{2}+y_{2}^{2}}{\nu}\right)^{-\frac{\nu+2}{2}}, \quad\left(y_{1}, y_{2}\right) \in \mathbb{R}^{2},
$$

and $\theta$ being the Pearson correlation coefficient. The stable tail dependence function can be described as

$$
L\left(y_{1}, y_{2} \mid \theta\right)=y_{2} F_{\nu+1}\left(\frac{\left(y_{2} / y_{1}\right)^{1 / \nu}-\theta}{\sqrt{1-\theta^{2}}} \sqrt{\nu+1}\right)+y_{1} F_{\nu+1}\left(\frac{\left(y_{1} / y_{2}\right)^{1 / \nu}-\theta}{\sqrt{1-\theta^{2}}} \sqrt{\nu+1}\right),
$$

where $F_{\nu+1}$ is the distribution function of the univariate Student distribution with $\nu+1$ degrees of freedom. Asymptotic independence can be reached for $\theta=-1$ and complete positive dependence for $\theta=1$. We set $\theta=X$, where $X$ is uniformly distributed on $[0,1]$. This model satisfies the second order condition with

$$
\begin{aligned}
M_{x}\left(y_{1}, y_{2}\right) & =C\left[y_{2}^{2 / \nu+1} F_{\nu+3}\left(\frac{\left(y_{2} / y_{1}\right)^{1 / \nu}-\theta}{\sqrt{1-\theta^{2}}} \sqrt{\nu+3}\right)+y_{1}^{2 / \nu+1} F_{\nu+3}\left(\frac{\left(y_{1} / y_{2}\right)^{1 / \nu}-\theta}{\sqrt{1-\theta^{2}}} \sqrt{\nu+3}\right)\right], \\
C & :=-\frac{\nu^{2 / \nu+1} \pi^{1 / \nu}(\nu+1)}{2(\nu+2)}\left(\frac{\Gamma\left(\frac{\nu}{2}\right)}{\Gamma\left(\frac{\nu+1}{2}\right)}\right)^{2 / \nu}, \\
\alpha_{x}(t) & =t^{-2 / \nu} .
\end{aligned}
$$

Moreover, one could check that the uniform property in the first and second order conditions are verified since we have continuity of the involved functions. The model satisfies also conditions $(\mathcal{D}),(\mathcal{L}),(\mathcal{A})$ and $\left(\mathcal{F}_{m}\right)$. In the simulations we set $\nu=1$.

- Model 2: We consider a particular case of the Archimax bivariate copulas introduced in Capéraà et al. (2000) and also mentioned in Fougères et al. (2015), namely:

$$
C\left(y_{1}, y_{2} \mid x\right)=\left\{1+L\left(y_{1}^{-1}-1, y_{2}^{-1}-1 \mid x\right)\right\}^{-1}
$$

where we use for $L$ the asymmetric logistic stable tail dependence function defined by

$$
L\left(y_{1}, y_{2} \mid x\right)=\left(1-t_{1}\right) y_{1}+\left(1-t_{2}\right) y_{2}+\left[\left(t_{1} y_{1}\right)^{\theta_{x}}+\left(t_{2} y_{2}\right)^{\theta_{x}}\right]^{1 / \theta_{x}},
$$

where $0 \leqslant t_{1}, t_{2} \leqslant 1$, and $\theta_{x}=\min (1 / x, 100)$, with the covariate $X$ uniformly distributed on $[0,1]$. The marginal distributions are taken to be unit Fréchet. This model satisfies our second 
order condition with

$$
\begin{aligned}
M_{x}\left(y_{1}, y_{2}\right) & =y_{1}^{2} \partial_{1} L\left(y_{1}, y_{2} \mid x\right)+y_{2}^{2} \partial_{2} L\left(y_{1}, y_{2} \mid x\right)-L^{2}\left(y_{1}, y_{2} \mid x\right), \\
\alpha_{x}(t) & =t^{-1},
\end{aligned}
$$

and also satisfies $(\mathcal{D}),(\mathcal{L}),(\mathcal{A})$ and $\left(\mathcal{F}_{m}\right)$. In the simulations, different values for the pair $\left(t_{1}, t_{2}\right)$ have been tried but the results seem to be not too much influenced by them, thus we exhibit only the results in case $\left(t_{1}, t_{2}\right)=(0.4,0.6)$ which corresponds to an asymmetric tail dependence function.

To compute our estimator $\bar{L}_{k}$, two sequences $h$ and $c$ have to be chosen. Concerning $c$, we can use the following cross validation criterion introduced by Yao (1999), implemented by Gannoun et al. (2002), and already used in an extreme value context by Daouia et al. (2011, 2013) or Goegebeur et al. (2015):

$$
c_{j}:=\arg \min _{c \in \mathcal{C}_{g}} \sum_{i=1}^{n} \sum_{k=1}^{n}\left[\mathbb{1}_{\left\{Y_{i}^{(j)} \leqslant Y_{k}^{(j)}\right\}}-\widetilde{F}_{n,-i, j}\left(Y_{k}^{(j)} \mid X_{i}\right)\right]^{2}, j=1,2,
$$

where $\mathcal{C}_{g}$ is a grid of values of $c$ and $\widetilde{F}_{n,-i, j}(y \mid x):=\frac{\sum_{k=1, k \neq i}^{n} K_{c}\left(x-X_{k}\right) \mathbb{1}_{\left\{Y_{k}^{(j)} \leqslant y\right\}}}{\sum_{k=1, k \neq i}^{n} K_{c}\left(x-X_{k}\right)}$. We select the sequence $h$ from the condition

$$
n \sqrt{\frac{h^{p}}{k}} \sqrt{\frac{\mid \log c^{q}}{n c^{p}}} \rightarrow 0
$$

by taking $h=c\left(\frac{k}{n}\right)^{1 / p}|\log c|^{-\xi}$, where $\xi p>q$ and $c:=\min \left(c_{1}, c_{2}\right)$.

For each distribution, we simulate $N=500$ samples of size $n=1000$, and we consider several positions $\left\{y_{t}:=(t / 10,1-t / 10) ; t=1, \ldots, 9\right\}$. Since all stable tail dependence functions satisfy $\max (t, 1-t) \leqslant L(t, 1-t) \leqslant 1$, all the estimators have been corrected so that they satisfy these bounds. However, the estimators have not been forced to be convex although this could have been done for instance by using a constrained spline smoothing method (Hall and Tajvidi, 2000), or a projection technique (Fils-Villetard et al., 2008). In all the settings, $\mathcal{C}_{g}=\{0.06,0.12,0.18,0.24,0.3\}$ and $\xi=1.1$ are used as chosen in Escobar-Bach et al. (2017a). An extensive simulation study has also indicated that these choices seem to give always reason- 
able results. Concerning the kernel, each time we use the bi-quadratic function

$$
K(x):=\frac{15}{16}\left(1-x^{2}\right)^{2} \mathbb{1}_{[-1,1]}(x) .
$$

As a qualitative measure of the efficiency over the different positions $\left\{y_{t}, t=1, \ldots, 9\right\}$, we define the absolute bias and the mean squared error (MSE) respectively as follows

$$
\begin{aligned}
\operatorname{Abias}(x, k) & :=\frac{1}{9 N} \sum_{t=1}^{9} \sum_{i=1}^{N}\left|\bar{L}_{k}^{(i)}\left(y_{t} \mid x\right)-L\left(y_{t} \mid x\right)\right| \\
\operatorname{MSE}(x, k) & :=\frac{1}{9 N} \sum_{t=1}^{9} \sum_{i=1}^{N}\left(\bar{L}_{k}^{(i)}\left(y_{t} \mid x\right)-L\left(y_{t} \mid x\right)\right)^{2} .
\end{aligned}
$$

Figures 1-3 (respectively Figures 4-6) represent the sample means in case of Model 1 (respectively Model 2), based on $N$ samples of size $n$, of our estimator $\bar{L}_{k}(y \mid x)$ as a function of $k$. Each of these figures shows the behaviour of our estimator at the positions $y \in\left\{y_{t}, t=1, \ldots, 9\right\}$ for a given value of the covariate $(x=0.2,0.5$ and 0.8 , respectively). Based on these simulations, we can conclude that in general, our estimator behaves well for not too large values of $k$ with a good proximity to the true value, while some bias appears for $k$ large, which can be expected from our theoretical results, since for $k$ large $\alpha_{x}(n / k)$ is not necessary negligible. The estimates obtained for the asymmetric logistic model show more bias than those for the bivariate Student distribution, since $\alpha_{x}(t)$ converges faster to zero as $t \rightarrow \infty$ for the latter. Indeed, for the bivariate Student distribution with $\nu=1$ we have $\alpha_{x}(t)=t^{-2}$ while $\alpha_{x}(t)=t^{-1}$ for the asymmetric logistic distribution. From the figures it also seems that the estimation is more difficult for $y$ close to the diagonal.

In Figures 7 and 8 we show the summary performance measures Abias and MSE for Model 1 and Model 2, respectively, as a function of $k$ for each of the covariate positions. As is clear from these figures, the performance measures do not critically depend on the position in the covariate space for $k$ not too large. The results also seem to indicate that the estimator performs better for stronger dependence than for weaker dependence.

\subsection{Application to air pollution data}

In this section, the proposed methodology is applied to a dataset of air pollution measurements. Being able to analyse the dependence between temperature and ozone concentration is of pri- 

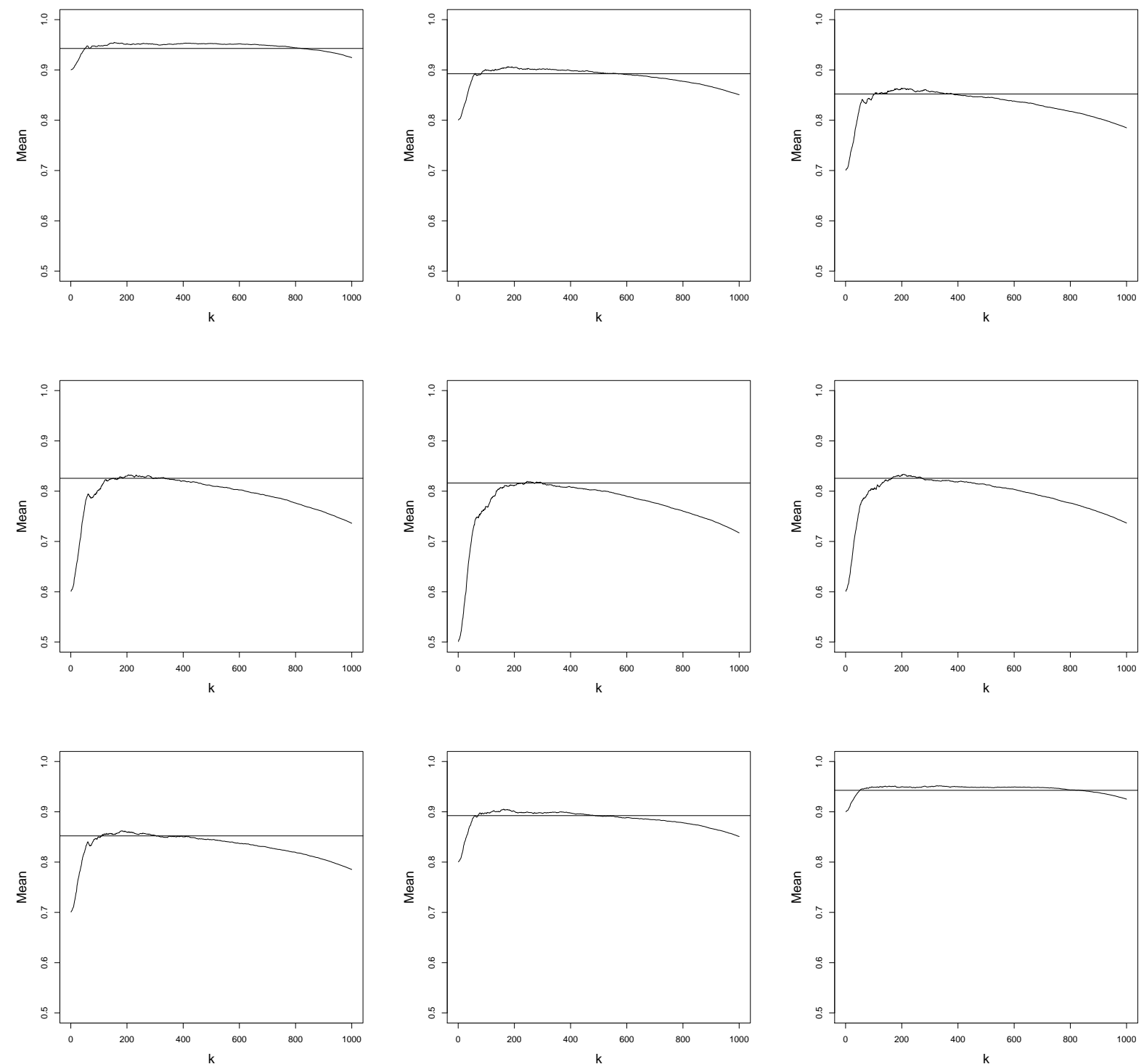

Figure 1: Model 1: Sample mean of $\bar{L}_{k}(y \mid 0.2)$ as a function of $k$ with, from left to right and up to down, $y=y_{t}, t=1, \ldots, 9$, respectively. The true value of the parameter is represented by a full horizontal black line. 

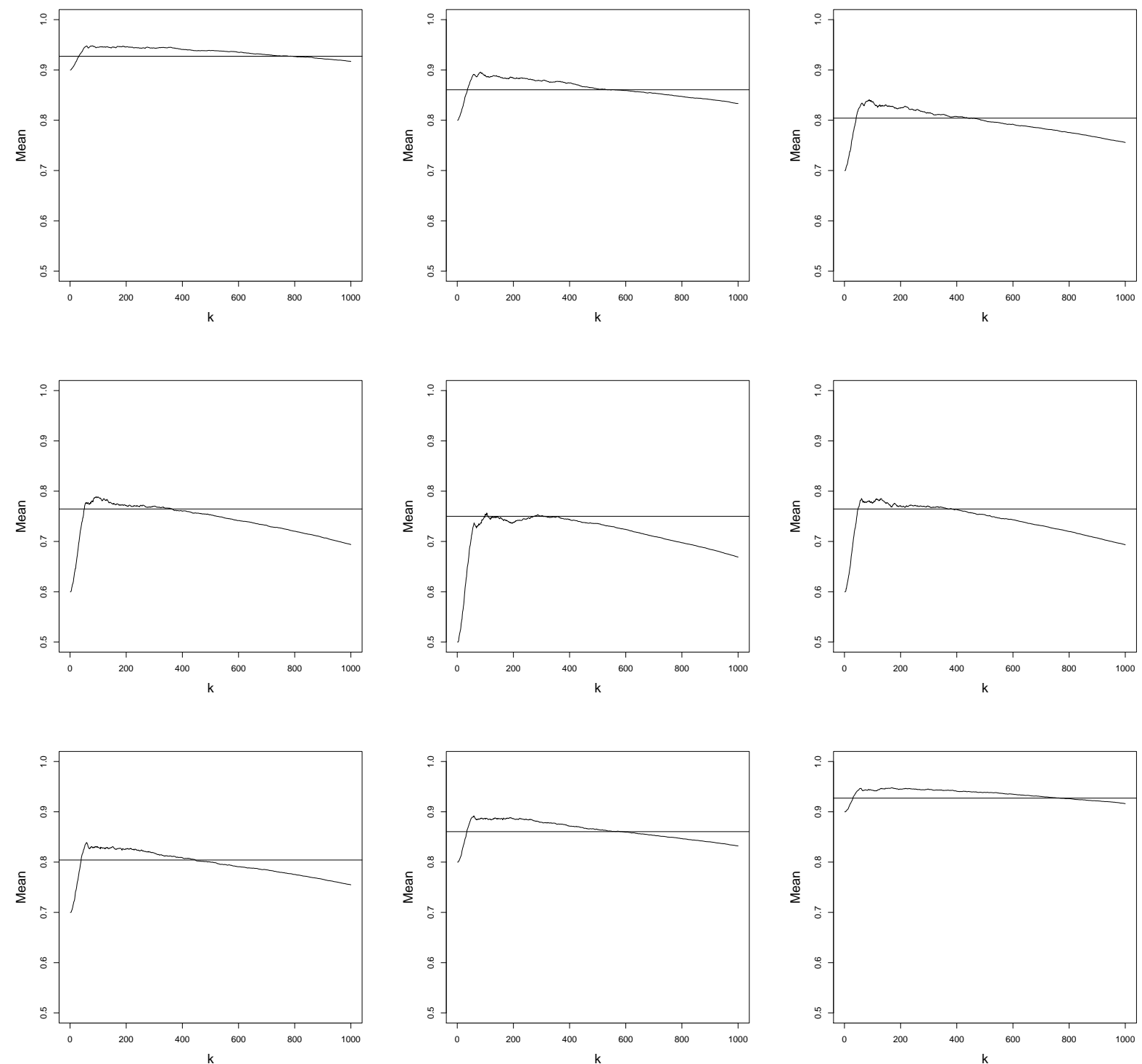

Figure 2: Model 1: Sample mean of $\bar{L}_{k}(y \mid 0.5)$ as a function of $k$ with, from left to right and up to down, $y=y_{t}, t=1, \ldots, 9$, respectively. The true value of the parameter is represented by a full horizontal black line. 

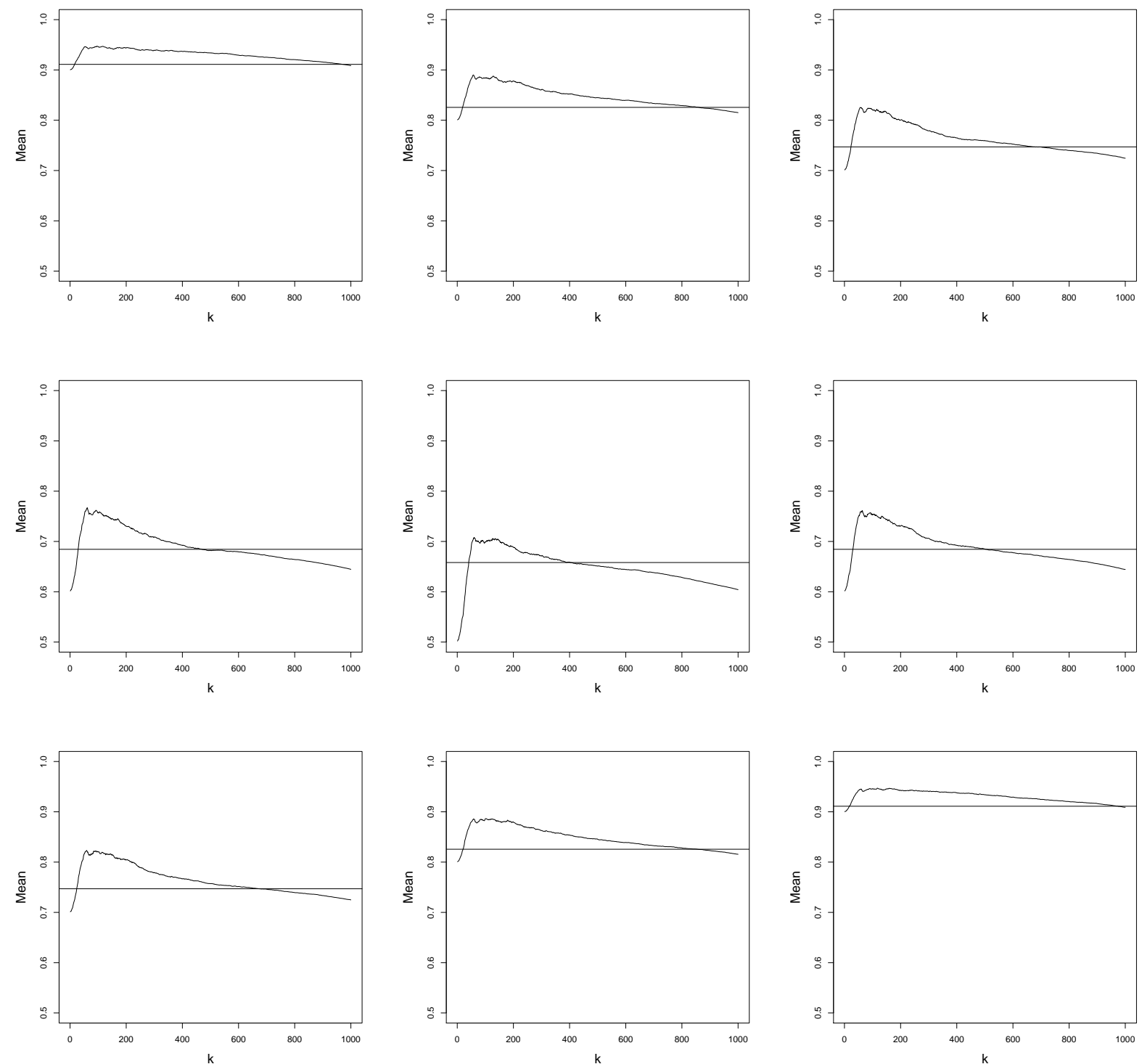

Figure 3: Model 1: Sample mean of $\bar{L}_{k}(y \mid 0.8)$ as a function of $k$ with, from left to right and up to down, $y=y_{t}, t=1, \ldots, 9$, respectively. The true value of the parameter is represented by a full horizontal black line. 

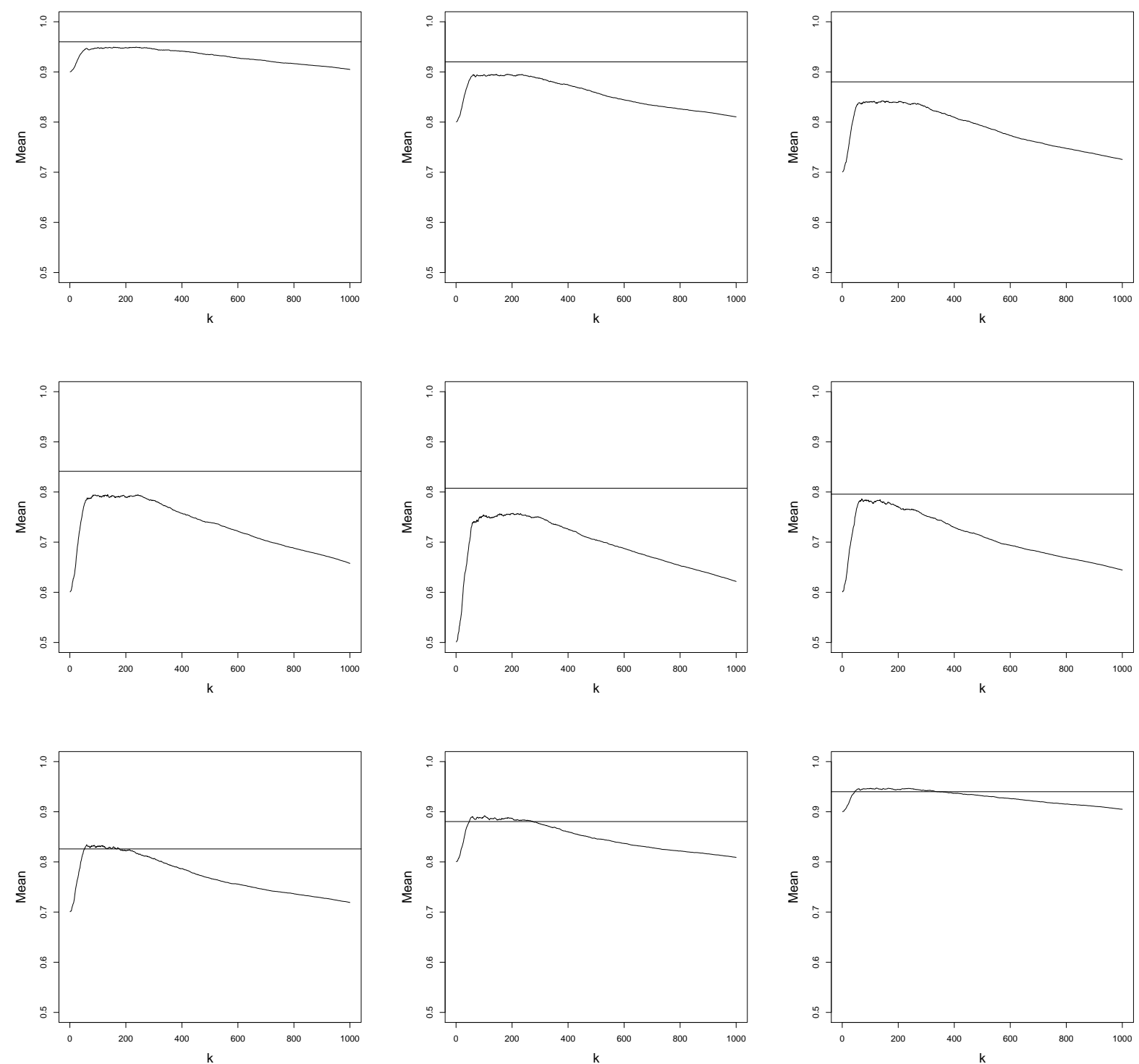

Figure 4: Model 2: Sample mean of $\bar{L}_{k}(y \mid 0.2)$ as a function of $k$ with, from left to right and up to down, $y=y_{t}, t=1, \ldots, 9$, respectively. The true value of the parameter is represented by a full horizontal black line. 

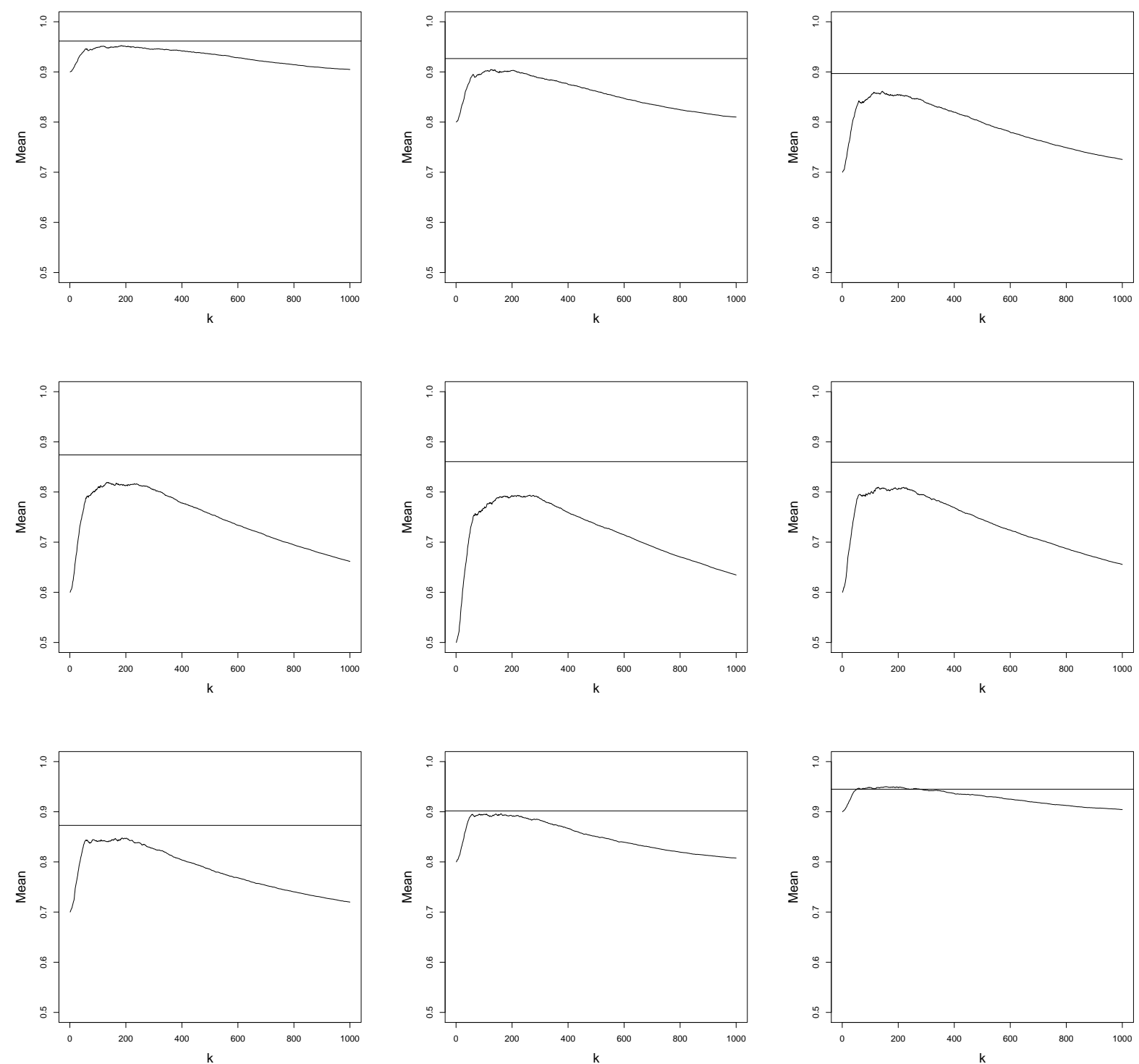

Figure 5: Model 2: Sample mean of $\bar{L}_{k}(y \mid 0.5)$ as a function of $k$ with, from left to right and up to down, $y=y_{t}, t=1, \ldots, 9$, respectively. The true value of the parameter is represented by a full horizontal black line. 

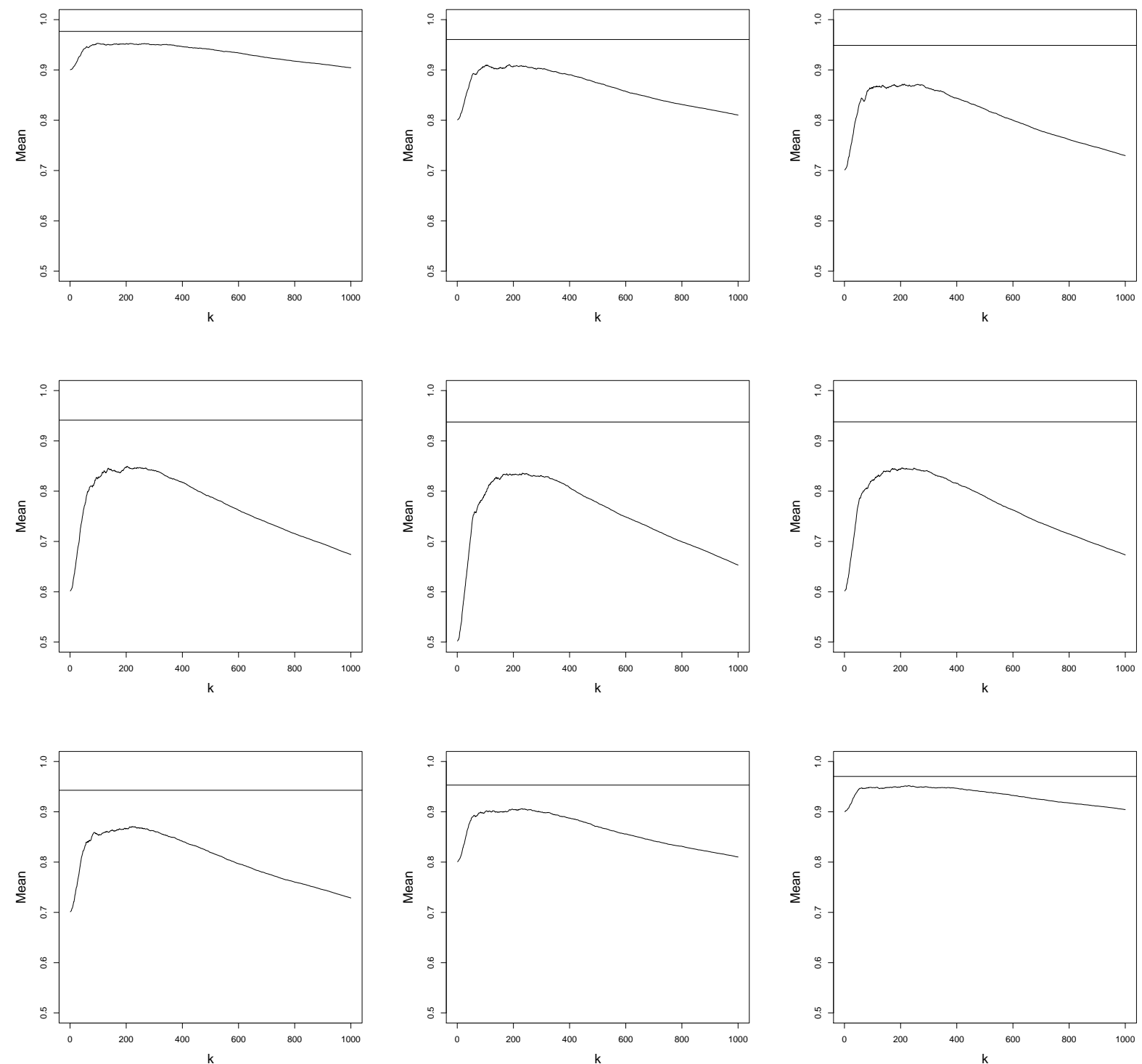

Figure 6: Model 2: Sample mean of $\bar{L}_{k}(y \mid 0.8)$ as a function of $k$ with, from left to right and up to down, $y=y_{t}, t=1, \ldots, 9$, respectively. The true value of the parameter is represented by a full horizontal black line. 

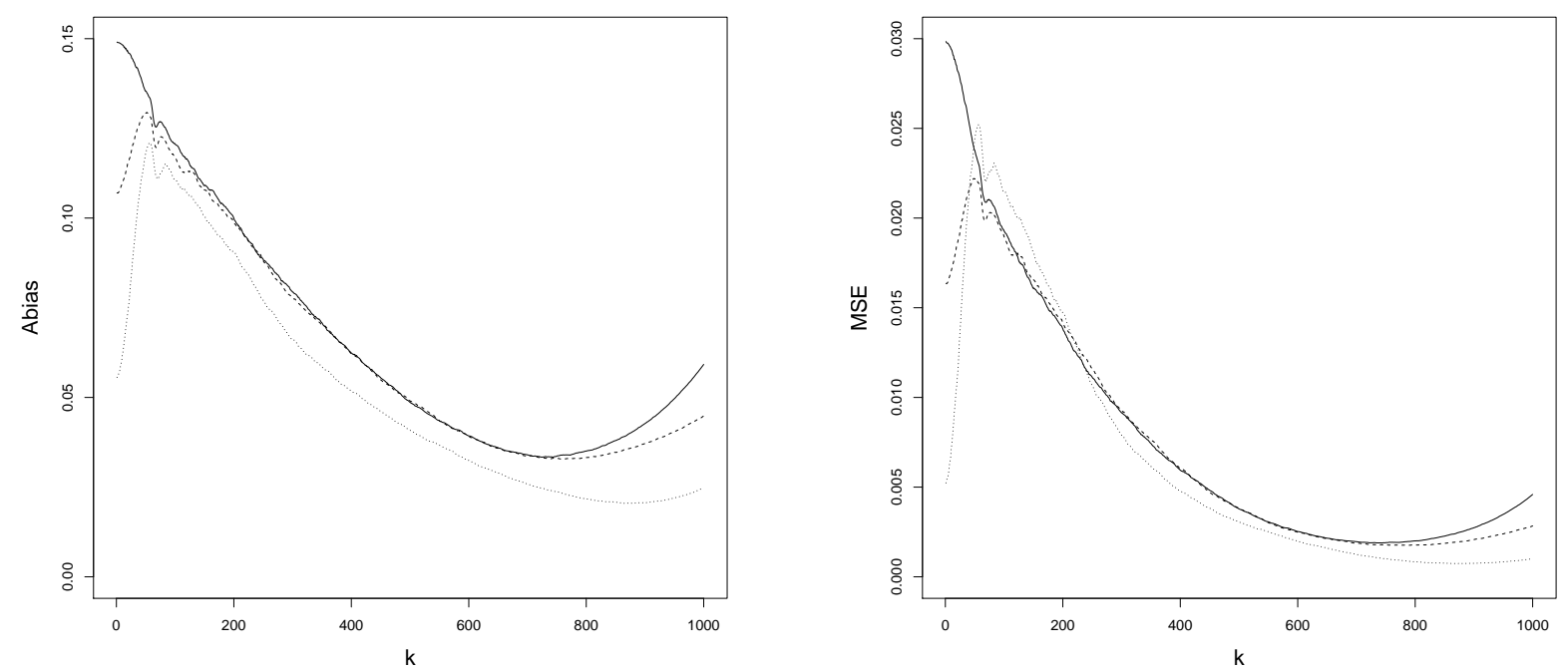

Figure 7: Model 1: Absolute bias and MSE as a function of $k$ for different covariate positions $x=0.2$ (full line), 0.5 (dashed line), 0.8 (dotted line).
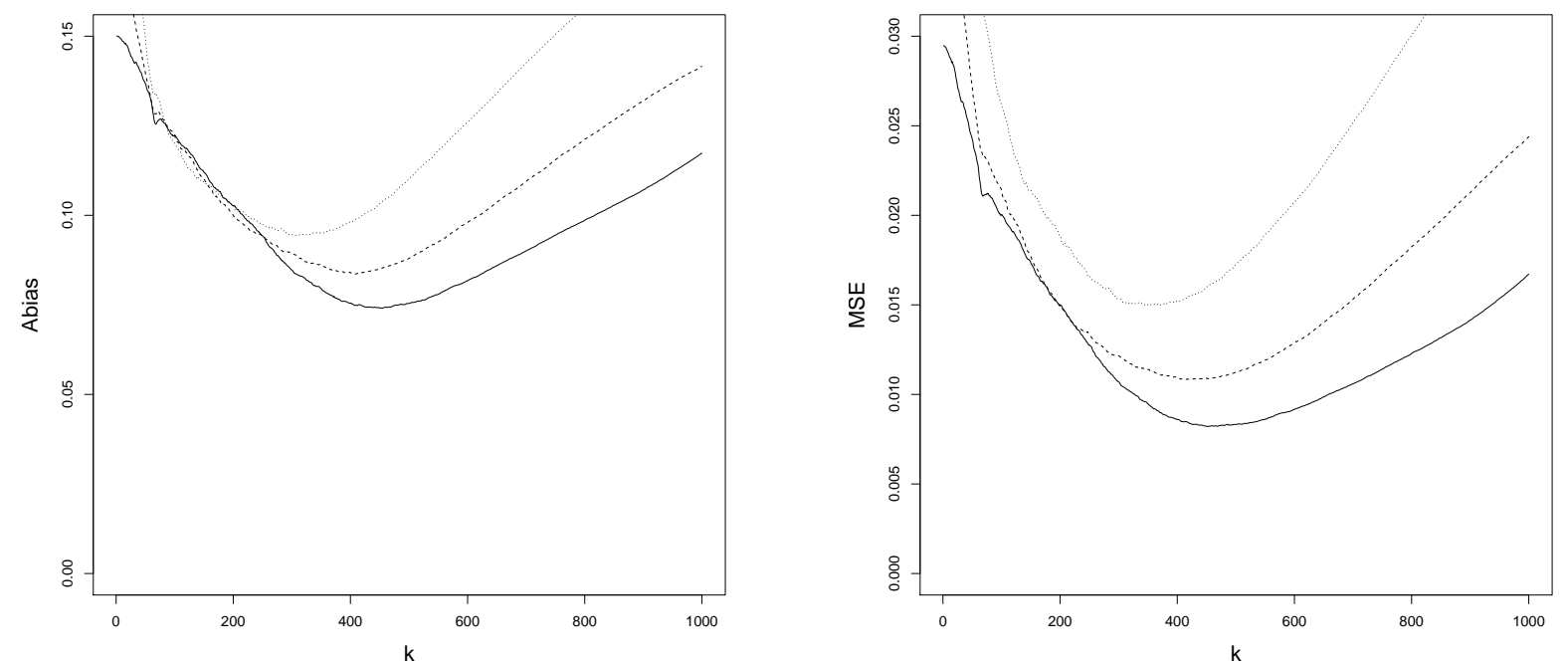

Figure 8: Model 2: Absolute bias and MSE as a function of $k$ for different covariate positions $x=0.2$ (full line), 0.5 (dashed line), 0.8 (dotted line). 
mary importance in order to identify population health effects of high ozone concentration and extreme temperature. The dataset contains daily measurements on, among others, maximum temperature and ground level ozone concentration, for the time period 1999 to 2013, collected at stations spread over the U.S. by the United States Environmental Protection Agency (EPA). It is publicly available at https://aqsdr1.epa.gov/aqsweb/aqstmp/airdata/download_files.html. We estimate the stable tail dependence function conditional on time and location, where the latter is expressed by latitude and longitude. The estimation method is the same as the one described in Section 3.1 apart from the dimension of the covariate space, namely here $p=3$ which implies $\xi=1.1 / 3$. We use the same grid values $\mathcal{C}_{g}$ for the cross-validation, after standardising the covariates to the interval $[0,1]$. As kernel function $K^{*}$ we use the following generalisation of the bi-quadratic kernel $K$ :

$$
K^{*}\left(x_{1}, x_{2}, x_{3}\right):=\prod_{i=1}^{3} K\left(x_{i}\right),
$$

where $x_{1}, x_{2}, x_{3}$, refer to the covariates time, latitude and longitude, respectively, in standardised form. Note that $K^{*}$ has as support the unit ball with respect to the max-norm on $\mathbb{R}^{3}$.

We report here only the results at two different time points: January 15, 2007 and June 15, 2007 in California. California has one of the largest economies in the world and as such there is a high emission of air pollutants. First, Figure 9 represents the stations in California as markers with different colors corresponding to the value of the estimates median $\left\{\bar{L}_{k}(0.5,0.5 \mid x), k=\right.$ $n / 4, \ldots, n / 2\}$ of $L(0.5,0.5 \mid x)$. The range over which the median is computed can be motivated from the simulations, see e.g. Figures 1 till 6 . Clearly, the extremal dependence between daily maximum temperature and ground level ozone concentration varies a lot across measurement stations. This could be explained by the fact that the climate of California varies widely, from hot desert to subarctic, depending on the location. As is also clear from Figure 9, the extremal dependence also varies over time. In order to get a better idea of the extremal dependence between temperature and ozone, we show in Figure 10 the time plot of the estimates of the conditional extremal coefficient $\eta(x):=2 \times L(0.5,0.5 \mid x) \in[1,2]$ at two specific stations, Fresno and Los Angeles. This coefficient is often used in the literature as a summary measure of the extremal dependence, with perfect dependence corresponding to the value 1 and independence 

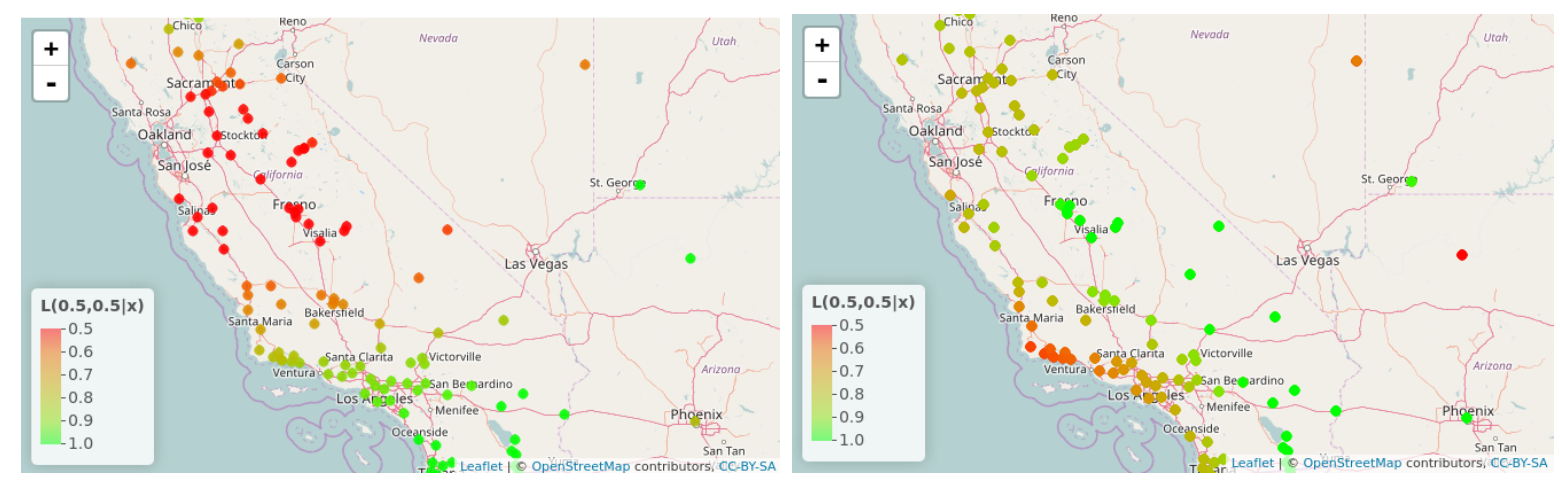

Figure 9: Air pollution data: Estimates median $\left\{\bar{L}_{k}(0.5,0.5 \mid x), k=n / 4, \ldots, n / 2\right\}$ of $L(0.5,0.5 \mid x)$ for stations in California on January 15, 2007 (left) and June 15, 2007 (right).

to the value 2. These two cities exhibit a different extremal dependence throughout the year. Indeed, for Fresno the extremal dependence is strong in the winter months but becomes weaker in summer, while the opposite holds for Los Angeles. To get a more detailed picture of the extremal dependence we show in Figure 11 the estimate median $\left\{\bar{L}_{k}(t, 1-t \mid x), k=n / 4, \ldots, n / 2\right\}$ of the Pickands dependence function for the cities Fresno (top row) and Los Angeles (bottom row) on January 15, 2007 (first column) and June 15, 2007 (second column). In Los Angeles the extremal dependence is stronger in summer than in spring and winter, which corresponds to the typical pattern (see e.g. Mahmud et al., 2008). Fresno deviates from this typical pattern, and the two variables are close to asymptotic independence during summer. This could be explained by the fact that ozone formation seems to be suppressed at extremely high temperatures, say above 312 Kelvin, due to different chemical and biophysical feedback mechanisms, and such temperature conditions are not unusual for the Central Valley of California; see Steiner et al. (2010). 

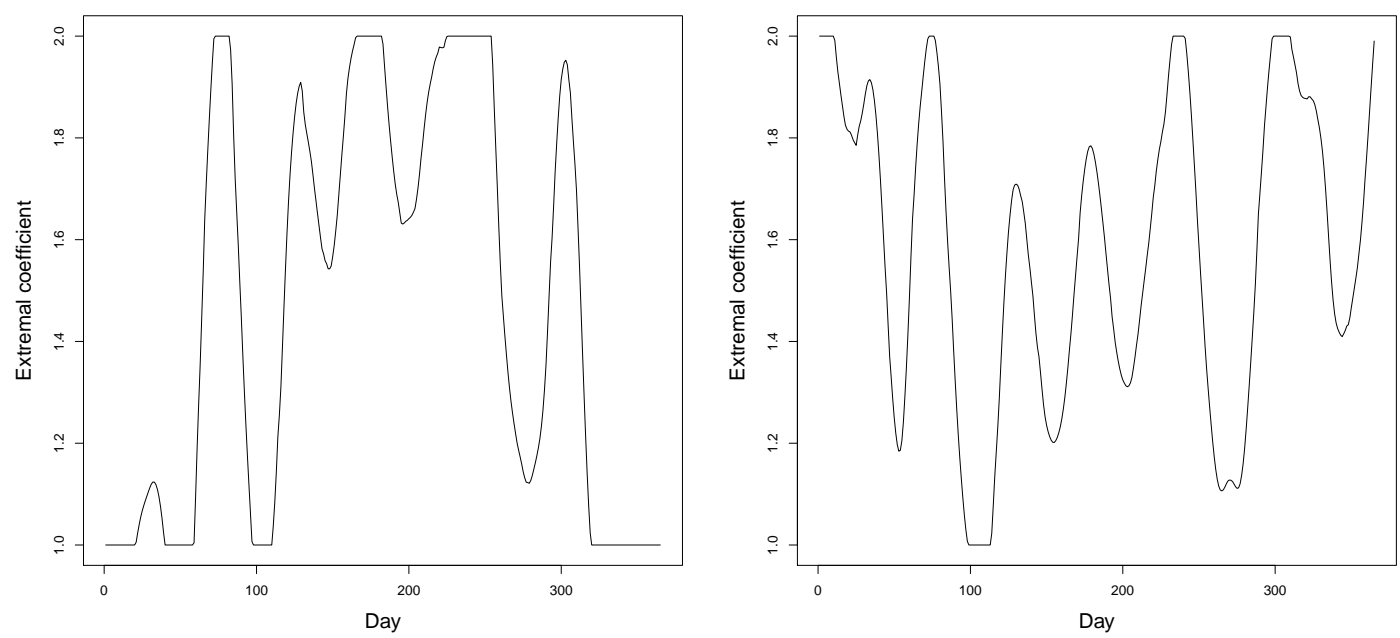

Figure 10: Air pollution data: Time plot of the estimate $2 \times \operatorname{median}\left\{\bar{L}_{k}(0.5,0.5 \mid x), k=\right.$ $n / 4, \ldots, n / 2\}$ of the conditional extremal coefficient at Fresno (left) and Los Angeles (right) over the year 2007 .

\section{Appendix: Proofs}

\subsection{Proof of Lemma 2.1}

In order to prove Lemma 2.1, we only need to verify that

$$
\mathbb{E}\left[\widehat{T}_{k}\left(y \mid x_{0}\right)\right] \rightarrow f\left(x_{0}\right) L\left(y \mid x_{0}\right) \quad \text { and } \quad \operatorname{Var}\left(\widehat{T}_{k}\left(y \mid x_{0}\right)\right) \rightarrow 0 \quad \text { as } n \rightarrow \infty
$$

We have

$$
\begin{aligned}
\mathbb{E}\left[\widehat{T}_{k}\left(y \mid x_{0}\right)\right] & =\int_{S_{K}} K(u) \frac{n}{k} \mathbb{P}\left(A_{n / k, y} \mid X=x_{0}-h u\right) f\left(x_{0}-h u\right) d u \\
& =\int_{S_{K}} K(u) L\left(y \mid x_{0}-h u\right) f\left(x_{0}-h u\right) d u \\
& +\int_{S_{K}} K(u)\left(\frac{n}{k} \mathbb{P}\left(A_{n / k, y} \mid X=x_{0}-h u\right)-L\left(y \mid x_{0}-h u\right)\right) f\left(x_{0}-h u\right) d u
\end{aligned}
$$

Since $u \in S_{K}$, for $n$ large enough, using the continuity of $f$ and $L$ at $x_{0} \in \operatorname{Int}\left(S_{X}\right)$ non-empty, we have boundedness in a neighborhood of $x_{0}$ and thus

$$
\sup _{u \in S_{K}} L\left(y \mid x_{0}-h u\right)<+\infty \text { and } \sup _{u \in S_{K}} f\left(x_{0}-h u\right)<+\infty,
$$



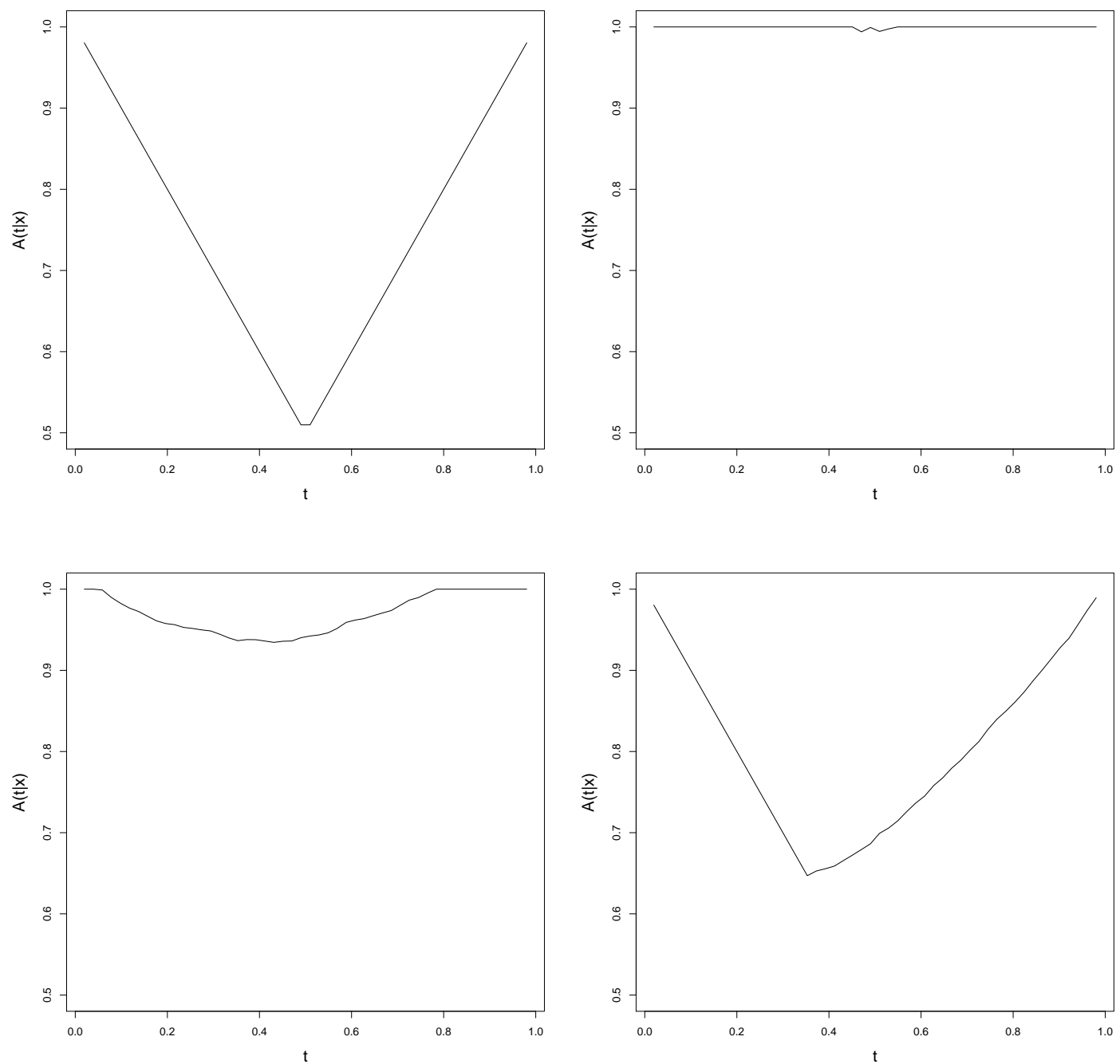

Figure 11: Air pollution data: Estimate of the Pickands dependence function median $\left\{\bar{L}_{k}(t, 1-\right.$ $t \mid x), k=n / 4, \ldots, n / 2\}$ for Fresno (top) and Los Angeles (bottom) on January 15, 2007 (first column) and June 15, 2007 (second column). 
and by the first order condition

$$
\sup _{u \in S_{K}}\left|\frac{n}{k} \mathbb{P}\left(A_{n / k, y} \mid X=x_{0}-h u\right)-L\left(y \mid x_{0}-h u\right)\right| \rightarrow 0,
$$

as $n \rightarrow \infty$.

Note that $\int K=1$, and hence by Lebesgue's dominated convergence theorem, we obtain, for $n \rightarrow \infty$,

$$
\int_{S_{K}} K(u) L\left(y \mid x_{0}-h u\right) f\left(x_{0}-h u\right) d u \rightarrow f\left(x_{0}\right) L\left(y \mid x_{0}\right),
$$

and

$$
\int_{S_{K}} K(u)\left(\frac{n}{k} \mathbb{P}\left(A_{n / k, y} \mid X=x_{0}-h u\right)-L\left(y \mid x_{0}-h u\right)\right) f\left(x_{0}-h u\right) d u \rightarrow 0,
$$

implying the first statement.

Next,

$$
\begin{aligned}
& \operatorname{Var}\left(\widehat{T}_{k}\left(y \mid x_{0}\right)\right) \\
= & \frac{1}{k}\left\{h^{-p} \int_{S_{K}} K^{2}(u) \frac{n}{k} \mathbb{P}\left(A_{n / k, y} \mid X=x_{0}-h u\right) f\left(x_{0}-h u\right) d u\right\}-\frac{1}{n}\left(f\left(x_{0}\right) L\left(y \mid x_{0}\right)+o(1)\right)^{2} \\
= & \frac{1}{k h^{p}}\left(\|K\|_{2}^{2} f\left(x_{0}\right) L\left(y \mid x_{0}\right)+o(1)\right)-\frac{1}{n}\left(f\left(x_{0}\right) L\left(y \mid x_{0}\right)+o(1)\right)^{2},
\end{aligned}
$$

which goes to zero since $k h^{p} \rightarrow \infty$.

\subsection{Proof of Lemma 2.2}

Clearly, we have

$$
\begin{aligned}
k h^{p} \operatorname{Cov}\left(\widehat{T}_{k}\left(y \mid x_{0}\right), \widehat{T}_{k}\left(y^{\prime} \mid x_{0}\right)\right) & =\int_{S_{K}} K^{2}(u) \frac{n}{k} \mathbb{P}\left(A_{n / k, y} \cap A_{n / k, y^{\prime}} \mid X=x_{0}-h u\right) f\left(x_{0}-h u\right) d u \\
& -h^{p} \frac{k}{n}\left(f\left(x_{0}\right)^{2} L\left(y \mid x_{0}\right) L\left(y^{\prime} \mid x_{0}\right)+o(1)\right) \\
& =\int_{S_{K}} K^{2}(u) \frac{n}{k} \mathbb{P}\left(A_{n / k, y} \cap A_{n / k, y^{\prime}} \mid X=x_{0}-h u\right) f\left(x_{0}-h u\right) d u+o(1) .
\end{aligned}
$$

Then, we easily deduce that

$$
\begin{aligned}
\mathbb{P}\left(A_{n / k, y} \cap A_{n / k, y^{\prime}} \mid X=x_{0}-h u\right) & =\mathbb{P}\left(A_{n / k, y} \mid X=x_{0}-h u\right)+\mathbb{P}\left(A_{n / k, y^{\prime}} \mid X=x_{0}-h u\right) \\
& -\mathbb{P}\left(A_{n / k, y} \cup A_{n / k, y^{\prime}} \mid X=x_{0}-h u\right) .
\end{aligned}
$$


Naturally we can describe the sets $A_{n / k, y}$ and $A_{n / k, y^{\prime}}$ as a finite union like for any $y \in \mathbb{R}_{+}^{d}$

$$
A_{n / k, y}=\bigcup_{j=1}^{d}\left\{1-F_{j}\left(Y^{(j)} \mid X\right) \leqslant \frac{k}{n} y_{j}\right\}=: \bigcup_{j=1}^{d} A_{n / k, y_{j}, j} .
$$

Thus, we have

$$
\begin{aligned}
A_{n / k, y} \cup A_{n / k, y^{\prime}} & =\bigcup_{j=1}^{d}\left\{A_{n / k, y_{j}, j} \cup A_{n / k, y_{j}^{\prime}, j}\right\} \\
& =\bigcup_{j=1}^{d}\left\{1-F_{j}\left(Y^{(j)} \mid X\right) \leqslant \frac{k}{n}\left(y_{j} \vee y_{j}^{\prime}\right)\right\} \\
& =\bigcup_{j=1}^{d} A_{n / k, y_{j} \vee y_{j}^{\prime}, j}=A_{n / k, y \vee y^{\prime}},
\end{aligned}
$$

which implies that

$$
\begin{aligned}
\mathbb{P}\left(A_{n / k, y} \cap A_{n / k, y^{\prime}} \mid X=x_{0}-h u\right) & =\mathbb{P}\left(A_{n / k, y} \mid X=x_{0}-h u\right)+\mathbb{P}\left(A_{n / k, y^{\prime}} \mid X=x_{0}-h u\right) \\
& -\mathbb{P}\left(A_{n / k, y \vee y^{\prime}} \mid X=x_{0}-h u\right),
\end{aligned}
$$

and using the same arguments as in the proof of Lemma 2.1, the result follows.

\subsection{Proof of Theorem 2.1}

As a first step we consider the process

$$
\left\{\sqrt{k h^{p}}\left(\widehat{T}_{k}\left(y \mid x_{0}\right)-f\left(x_{0}\right)\left[L\left(y \mid x_{0}\right)+\alpha_{x_{0}}\left(\frac{n}{k}\right) M_{x_{0}}(y)\right]\right), y \in[0, T]^{d}\right\}
$$

and study its weak convergence. Based on the decomposition

$$
\begin{aligned}
\sqrt{k h^{p}} & \left(\widehat{T}_{k}\left(y \mid x_{0}\right)-f\left(x_{0}\right)\left[L\left(y \mid x_{0}\right)+\alpha_{x_{0}}\left(\frac{n}{k}\right) M_{x_{0}}(y)\right]\right) \\
= & \sqrt{k h^{p}}\left(\widehat{T}_{k}\left(y \mid x_{0}\right)-\mathbb{E}\left[\widehat{T}_{k}\left(y \mid x_{0}\right)\right]\right) \\
& +\sqrt{k h^{p}}\left(\mathbb{E}\left[\widehat{T}_{k}\left(y \mid x_{0}\right)\right]-f\left(x_{0}\right)\left[L\left(y \mid x_{0}\right)+\alpha_{x_{0}}\left(\frac{n}{k}\right) M_{x_{0}}(y)\right]\right),
\end{aligned}
$$

we have that the main task is to study the weak convergence of the process

$$
\left\{\sqrt{k h^{p}}\left(\widehat{T}_{k}\left(y \mid x_{0}\right)-\mathbb{E}\left[\widehat{T}_{k}\left(y \mid x_{0}\right)\right]\right), y \in[0, T]^{d}\right\}
$$


since

$$
\lim _{n \rightarrow \infty} \sup _{y \in[0, T]^{d}} \sqrt{k h^{p}}\left|\mathbb{E}\left[\widehat{T}_{k}\left(y \mid x_{0}\right)\right]-f\left(x_{0}\right)\left[L\left(y \mid x_{0}\right)+\alpha_{x_{0}}\left(\frac{n}{k}\right) M_{x_{0}}(y)\right]\right|=0
$$

Indeed, if we look at (6) in the proof of Lemma 2.1

$$
\begin{aligned}
\mathbb{E}\left[\widehat{T}_{k}\left(y \mid x_{0}\right)\right] & =f\left(x_{0}\right) L\left(y \mid x_{0}\right)+O\left(h^{\eta_{f} \wedge \eta_{L}}\right) \\
& +\int_{S_{K}} K(u)\left(\frac{n}{k} \mathbb{P}\left(A_{n / k, y} \mid X=x_{0}-h u\right)-L\left(y \mid x_{0}-h u\right)\right) f\left(x_{0}-h u\right) d u,
\end{aligned}
$$

where the big $O$ term is independent from $y$. Then

$$
\begin{aligned}
& \frac{n}{k} \mathbb{P}\left(A_{n / k, y} \mid X=x_{0}-h u\right)-L\left(y \mid x_{0}-h u\right) \\
= & \alpha_{x_{0}-h u}\left(\frac{n}{k}\right)\left\{M_{x_{0}-h u}(y)+\left[\frac{\frac{n}{k} \mathbb{P}\left(A_{n / k, y} \mid X=x_{0}-h u\right)-L\left(y \mid x_{0}-h u\right)}{\alpha_{x_{0}-h u}(n / k)}-M_{x_{0}-h u}(y)\right]\right\},
\end{aligned}
$$

where

$$
\begin{aligned}
& \sup _{y \in[0, T]^{d}}\left|\frac{\frac{n}{k} \mathbb{P}\left(A_{n / k, y} \mid X=x_{0}-h u\right)-L\left(y \mid x_{0}-h u\right)}{\alpha_{x_{0}-h u}(n / k)}-M_{x_{0}-h u}(y)\right| \\
& \leqslant \quad \sup _{y \in[0, T]^{d}, x \in B_{x_{0}}(r)}\left|\frac{\frac{n}{k} \mathbb{P}\left(A_{n / k, y} \mid X=x\right)-L(y \mid x)}{\alpha_{x}(n / k)}-M_{x}(y)\right| \rightarrow 0,
\end{aligned}
$$

combining the second order condition with the fact that for $n$ large enough $x_{0}-h u \in B_{x_{0}}(r)$.

This leads to

$$
\begin{aligned}
& \frac{n}{k} \mathbb{P}\left(A_{n / k, y} \mid X=x_{0}-h u\right)-L\left(y \mid x_{0}-h u\right) \\
= & \alpha_{x_{0}-h u}\left(\frac{n}{k}\right)\left(M_{x_{0}-h u}(y)+o(1)\right) \\
= & \alpha_{x_{0}}\left(\frac{n}{k}\right)\left(M_{x_{0}-h u}(y)+o(1)\right)+\left(\alpha_{x_{0}-h u}\left(\frac{n}{k}\right)-\alpha_{x_{0}}\left(\frac{n}{k}\right)\right)\left(M_{x_{0}-h u}(y)+o(1)\right),
\end{aligned}
$$

where the little $o$ component doesn't depend on $y$. Now,

- according to the Hölder condition on $\alpha$ and $\|u\| \leqslant 1$

$$
\sup _{t \geqslant 0}\left|\alpha_{x_{0}-h u}(t)-\alpha_{x_{0}}(t)\right| \leqslant M_{\alpha}\|h u\|^{\eta_{\alpha}}=O\left(h^{\eta_{\alpha}}\right)
$$


- by uniform continuity of $(x, y) \rightarrow M_{x}(y)$ over $B_{x_{0}}(r) \times[0, T]^{d}$

$$
\sup _{y \in[0, T]^{d}}\left|M_{x_{0}-h u}(y)-M_{x_{0}}(y)\right| \rightarrow 0 \text { as } n \rightarrow \infty .
$$

Hence, we can deduce that

$$
\frac{n}{k} \mathbb{P}\left(A_{n / k, y} \mid X=x_{0}-h u\right)-L\left(y \mid x_{0}-h u\right)=\alpha_{x_{0}}\left(\frac{n}{k}\right) M_{x_{0}}(y)+\alpha_{x_{0}}\left(\frac{n}{k}\right) o(1)+O\left(h^{\eta_{\alpha}}\right),
$$

which implies that

$$
\begin{aligned}
& \sqrt{k h^{p}}\left|\mathbb{E}\left[\widehat{T}_{k}\left(y \mid x_{0}\right)\right]-f\left(x_{0}\right)\left[L\left(y \mid x_{0}\right)+\alpha_{x_{0}}\left(\frac{n}{k}\right) M_{x_{0}}(y)\right]\right| \\
= & O\left(\sqrt{k h^{p}} h^{\min \left(\eta_{f}, \eta_{L}, \eta_{\alpha}\right)}\right)+\sqrt{k h^{p}} \alpha_{x_{0}}\left(\frac{n}{k}\right) o(1) \rightarrow 0 .
\end{aligned}
$$

Define now the covering number $N\left(\mathcal{F}, L_{2}(Q), \tau\right)$ as the minimal number of $L_{2}(Q)$-balls of radius $\tau$ needed to cover the class of functions $\mathcal{F}$ and the uniform entropy integral as

$$
J\left(\delta, \mathcal{F}, L_{2}\right):=\int_{0}^{\delta} \sqrt{\log \sup _{Q \in \mathcal{Q}} N\left(\mathcal{F}, L_{2}(Q), \tau\|F\|_{Q, 2}\right)} d \tau,
$$

where $\mathcal{Q}$ is the set of all probability measures $Q$ for which $0<\|F\|_{Q, 2}^{2}:=\int F^{2} d Q<\infty$ and $F$ is an envelope function for the class $\mathcal{F}$.

Let $P$ be the distribution measure of $(Y, X)$, and denote the expected value under $P$, the empirical version and empirical process as follows

$$
P f:=\int f d P, \quad \mathbb{P}_{n} f:=\frac{1}{n} \sum_{i=1}^{n} f\left(Y_{i}, X_{i}\right), \quad \mathbb{G}_{n} f:=\sqrt{n}\left(\mathbb{P}_{n}-P\right) f,
$$

for any real-valued measurable function $f: \mathbb{R}^{d} \times \mathbb{R}^{p} \rightarrow \mathbb{R}$.

We introduce our sequence of classes $\mathcal{F}_{n}$ on $\mathbb{R}^{d} \times \mathbb{R}^{p}$ as

$$
\mathcal{F}_{n}:=\left\{(u, z) \rightarrow f_{n, y}(u, z), y \in[0, T]^{d}\right\}
$$

where

$$
f_{n, y}(u, z):=\sqrt{\frac{n}{k} h^{p}} K_{h}\left(x_{0}-z\right) \mathbb{1}_{\left\{1-F_{1}\left(u_{1} \mid z\right) \leqslant k / n y_{1} \text { or } \ldots \text { or } 1-F_{d}\left(u_{d} \mid z\right) \leqslant k / n y_{d}\right\}} .
$$


Denote also by $F_{n}$ an envelope function of the class $\mathcal{F}_{n}$. Now, according to Theorem 19.28 in van der Vaart (1998), the weak convergence of the stochastic process (7) follows from the following four conditions. Let $\rho_{x_{0}}$ be a semimetric, possibly depending on $x_{0}$, making $[0, T]^{d}$ totally bounded. We have to prove that

$$
\begin{aligned}
\sup _{\rho_{x_{0}}\left(y, y^{\prime}\right) \leqslant \delta_{n}} P\left(f_{n, y}-f_{n, y^{\prime}}\right)^{2} & \longrightarrow 0 \text { for every } \delta_{n} \searrow 0, \\
P F_{n}^{2} & =O(1), \\
P F_{n}^{2} 1_{\left\{F_{n}>\varepsilon \sqrt{n}\right\}} & \longrightarrow 0 \text { for every } \varepsilon>0, \\
J\left(\delta_{n}, \mathcal{F}_{n}, L_{2}\right) & \longrightarrow 0 \text { for every } \delta_{n} \searrow 0 .
\end{aligned}
$$

We start with proving (8). We have

$$
P\left(f_{n, y}-f_{n, y^{\prime}}\right)^{2}=\int_{S_{K}} K(u)^{2} \frac{n}{k} \mathbb{E}\left[\left(1_{A_{n / k, y}}-\mathbb{1}_{A_{n / k, y^{\prime}}}\right)^{2} \mid X=x_{0}-h u\right] f\left(x_{0}-h u\right) d u .
$$

But, for any $x^{\prime} \in S_{X}$

$$
\begin{aligned}
\mathbb{E}\left[\left(\mathbb{1}_{A_{n / k, y}}-\mathbb{1}_{A_{n / k, y^{\prime}}}\right)^{2} \mid X=x^{\prime}\right] & =\mathbb{P}\left(A_{n / k, y} \cup A_{n / k, y^{\prime}} \mid X=x^{\prime}\right)-\mathbb{P}\left(A_{n / k, y} \cap A_{n / k, y^{\prime}} \mid X=x^{\prime}\right) \\
& =\mathbb{P}\left(\left\{A_{n / k, y} \cup A_{n / k, y^{\prime}}\right\} \backslash\left\{A_{n / k, y} \cap A_{n / k, y^{\prime}}\right\} \mid X=x^{\prime}\right) .
\end{aligned}
$$

Using the same notation as in the proof of Lemma 2.2, we have

$$
\begin{aligned}
A_{n / k, y} \cap A_{n / k, y^{\prime}} & =\left\{\bigcup_{j=1}^{d} A_{n / k, y_{j}, j}\right\} \cap\left\{\bigcup_{j=1}^{d} A_{n / k, y_{j}^{\prime}, j}\right\} \\
& =\bigcup_{j=1}^{d} \bigcup_{i=1}^{d}\left\{A_{n / k, y_{j}, j} \cap A_{n / k, y_{i}^{\prime}, i}\right\} \\
& \supset \bigcup_{j=1}^{d}\left\{A_{n / k, y_{j}, j} \cap A_{n / k, y_{j}^{\prime}, j}\right\} \\
& =\bigcup_{j=1}^{d} A_{n / k, y_{j} \wedge y_{j}^{\prime}, j} .
\end{aligned}
$$


Then, with $A^{c}$ denoting the complement of any set $A$, it follows that

$$
\begin{aligned}
\left\{A_{n / k, y} \cup A_{n / k, y^{\prime}}\right\}\left\{A_{n / k, y} \cap A_{n / k, y^{\prime}}\right\} & \subset\left\{\bigcup_{j=1}^{d} A_{n / k, y_{j} \vee y_{j}^{\prime}, j}\right\} \bigcap\left\{\bigcap_{j=1}^{d} A_{n / k, y_{j} \wedge y_{j}^{\prime}, j}^{c}\right\} \\
& \subset \bigcup_{j=1}^{d}\left\{A_{n / k, y_{j} \vee y_{j}^{\prime}, j} \cap A_{n / k, y_{j} \wedge y_{j}^{\prime}, j}^{c}\right\} \\
& =\bigcup_{j=1}^{d}\left\{\frac{k}{n}\left(y_{j} \wedge y_{j}^{\prime}\right) \leqslant 1-F_{j}\left(Y^{(j)} \mid X\right) \leqslant \frac{k}{n}\left(y_{j} \vee y_{j}^{\prime}\right)\right\} .
\end{aligned}
$$

Returning now to (12), we have

$$
\begin{aligned}
& \mathbb{P}\left(\left\{A_{n / k, y} \cup A_{n / k, y^{\prime}}\right\} \backslash\left\{A_{n / k, y} \cap A_{n / k, y^{\prime}}\right\} \mid X=x^{\prime}\right) \\
\leqslant & \sum_{j=1}^{d} \mathbb{P}\left(\frac{k}{n}\left(y_{j} \wedge y_{j}^{\prime}\right) \leqslant 1-F_{j}\left(Y^{(j)} \mid X\right) \leqslant \frac{k}{n}\left(y_{j} \vee y_{j}^{\prime}\right) \mid X=x^{\prime}\right) \leqslant \frac{k}{n} \sum_{j=1}^{d}\left|y_{j}-y_{j}^{\prime}\right| .
\end{aligned}
$$

Thus, defining

$$
\rho_{x_{0}}\left(y, y^{\prime}\right)=\sum_{j=1}^{d}\left|y_{j}-y_{j}^{\prime}\right|,
$$

also called the Manhattan distance on $\mathbb{R}^{d}$, which is clearly a semimetric making $[0, T]^{d}$ totally bounded, we have proven (8).

We define now the envelope functions

$$
F_{n}(u, z):=\sqrt{\frac{n}{k} h^{p}} K_{h}\left(x_{0}-z\right) \mathbb{1}_{\left\{1-F_{1}\left(u_{1} \mid z\right) \leqslant k / n T \text { or } \ldots \text { or } 1-F_{d}\left(u_{d} \mid z\right) \leqslant k / n T\right\}} .
$$

With $y_{T}:=\underbrace{(T, \ldots, T)}_{d \text { times }}$, assertion (9) results from Lemma 2.1 since

$$
\begin{aligned}
P F_{n}^{2} & =\int_{S_{K}} K(u)^{2} \frac{n}{k} \mathbb{P}\left(A_{n / k, y_{T}} \mid X=x_{0}-h u\right) f\left(x_{0}-h u\right) d u \\
& =L\left(y_{T} \mid x_{0}\right) f\left(x_{0}\right)\|K\|_{2}^{2}+o(1) .
\end{aligned}
$$

For (10), note that we have $\left\{F_{n}>\varepsilon \sqrt{n}\right\}=\left\{\left(F_{n} / \varepsilon \sqrt{n}\right)^{\xi}>1\right\}$ for any $\xi>0$, thus

$$
\begin{aligned}
P F_{n}^{2} \mathbb{1}_{\left\{F_{n}>\varepsilon \sqrt{n}\right\}} & \leqslant \frac{1}{\varepsilon^{\xi} n^{\xi / 2}} P F_{n}^{2+\xi} \\
& =\frac{1}{\varepsilon^{\xi} n^{\xi / 2}}\left(\frac{n}{k h^{p}}\right)^{\xi / 2} \int_{S_{K}} K(u)^{2+\xi} \frac{n}{k} \mathbb{P}\left(A_{n / k, y_{T}} \mid X=x_{0}-h u\right) f\left(x_{0}-h u\right) d u \\
& =\left(\varepsilon \sqrt{k h^{p}}\right)^{-\xi}\left(\|K\|_{2+\xi}^{2+\xi} L\left(y_{T} \mid x_{0}\right) f\left(x_{0}\right)+o(1)\right),
\end{aligned}
$$


where the right-hand side converges towards 0 since $k h^{p} \rightarrow \infty$ and $K$ satisfies Assumption $\left(\mathcal{K}_{1}\right)$.

Finally, it remains to prove (11). Define the following class of functions on $\mathbb{R}^{d} \times \mathbb{R}^{p}$

$$
\begin{aligned}
\tilde{\mathcal{F}} & :=\left\{(u, z) \rightarrow \mathbb{1}_{\left\{1-F_{1}\left(u_{1} \mid z\right) \leqslant y_{1} \text { or } \ldots \text { or } 1-F_{d}\left(u_{d} \mid z\right) \leqslant y_{d}\right\}}, y \in \mathbb{R}_{+}^{d}\right\} \\
& =\left\{(u, z) \rightarrow \mathbb{1}_{\left\{1-\cdot \leqslant y_{1} \text { or } \ldots \text { or } 1-\cdot \leqslant y_{d}\right\}} \circ\left(F_{1}, \ldots, F_{d}\right)(u, z), y \in \mathbb{R}_{+}^{d}\right\} .
\end{aligned}
$$

Let's focus for a moment on the class of functions on $[0,1]^{d}$

$$
\left\{u \rightarrow \mathbb{1}_{\left\{1-u_{1} \leqslant y_{1} \text { or } \ldots \text { or } 1-u_{d} \leqslant y_{d}\right\}}, \quad y \in \mathbb{R}_{+}^{d}\right\} \text {. }
$$

Since this is a family of indicator functions, it is a $V C$-class if and only if the family of sets associated to the indicator functions is a $V C$-class of sets. The latter sets can be easily represented as the union of $d V C$-classes of sets and thus it is also a $V C$-class of sets (see Lemma 2.6.17 (iii) in van der Vaart and Wellner, 1996). Next, according to Lemma 2.6.18 (vii) in van der Vaart and Wellner (1996), it follows that $\tilde{\mathcal{F}}$ is a $V C$-class with $V C$-index $V$ fixed. Define now

$$
\tilde{\mathcal{F}}_{n}:=\left\{(u, z) \rightarrow \mathbb{1}_{\left\{1-F_{1}\left(u_{1} \mid z\right) \leqslant k / n y_{1} \text { or } \ldots \text { or } 1-F_{d}\left(u_{d} \mid z\right) \leqslant k / n y_{d}\right\}}, y \in[0, T]^{d}\right\},
$$

and the envelope function $\widetilde{F}_{n}(u, z):=\mathbb{1}_{\left\{1-F_{1}\left(u_{1} \mid z\right) \leqslant k / n T \text { or } \ldots \text { or } 1-F_{d}\left(u_{d} \mid z\right) \leqslant k / n T\right\}}$. The previous arguments for $\tilde{\mathcal{F}}$ remain, thus we have that $\tilde{\mathcal{F}}_{n}$ is also a $V C$-class with $V C$-index $V$. According to Theorem 2.6.7 in van der Vaart and Wellner (1996), there exists a universal constant $C$ such that for $\mathcal{Q}$ the set of all probability measures on $\mathbb{R}^{d} \times \mathbb{R}^{p}$ and any $0<\tau<1$

$$
\sup _{Q \in \mathcal{Q}} N\left(\tilde{\mathcal{F}}_{n}, L_{2}(Q), \tau\left\|\widetilde{F}_{n}\right\|_{\mathcal{Q}, 2}\right) \leqslant C V(16 e)^{V}\left(\frac{1}{\tau}\right)^{2(V-1)} .
$$

Next, we retrieve $\mathcal{F}_{n}$ by multiplying the previous family with one single function, i.e.

$$
\mathcal{F}_{n}=\left\{z \rightarrow \sqrt{n h^{p} / k} K_{h}\left(x_{0}-z\right)\right\} \times \tilde{\mathcal{F}}_{n}
$$

Since only one ball is needed to cover the class $\left\{z \rightarrow \sqrt{n h^{p} / k} K_{h}\left(x_{0}-z\right)\right\}$ whatever the measure $Q \in \mathcal{Q}$, according to the last inequality in the proof of Theorem 2.10.20 in van der Vaart and Wellner (1996)

$$
\sup _{Q \in \mathcal{Q}} N\left(\mathcal{F}_{n}, L_{2}(Q), \tau\left\|F_{n}\right\|_{Q, 2}\right) \leqslant C V(16 e)^{V}\left(\frac{1}{\tau}\right)^{2(V-1)}:=L\left(\frac{1}{\tau}\right)^{V} .
$$


Thus, (11) is established since for any sequence $\delta_{n} \searrow 0$ and $n$ large enough, we have

$$
J\left(\delta_{n}, \mathcal{F}_{n}, L_{2}\right) \leqslant \int_{0}^{\delta_{n}} \sqrt{\log (L)-V \log (\tau)} d \tau=o(1) .
$$

Finally, we consider the process (3). Straightforward calculations give the following decomposition

$$
\begin{aligned}
\sqrt{k h^{p}} & \left(\frac{\widehat{T}_{k}\left(y \mid x_{0}\right)}{\widehat{f}_{n}\left(x_{0}\right)}-L\left(y \mid x_{0}\right)-\alpha_{x_{0}}\left(\frac{n}{k}\right) M_{x_{0}}(y)\right) \\
= & \frac{\sqrt{k h^{p}}}{f\left(x_{0}\right)}\left(\widehat{T}_{k}\left(y \mid x_{0}\right)-f\left(x_{0}\right)\left[L\left(y \mid x_{0}\right)+\alpha_{x_{0}}\left(\frac{n}{k}\right) M_{x_{0}}(y)\right]\right)-\sqrt{k h^{p}} \frac{\widehat{T}_{k}\left(y \mid x_{0}\right)}{\hat{f}_{n}\left(x_{0}\right) f\left(x_{0}\right)}\left(\widehat{f}_{n}\left(x_{0}\right)-f\left(x_{0}\right)\right) .
\end{aligned}
$$

Note that for the first term in the right-hand side of the above display we have just established the weak convergence as a stochastic process (apart from the factor $1 / f\left(x_{0}\right)$ ), whereas for the second term we have essentially to study $\sqrt{k h^{p}}\left(\hat{f}_{n}\left(x_{0}\right)-f\left(x_{0}\right)\right)$. The latter can be rewritten as

$$
\sqrt{k h^{p}}\left(\widehat{f}_{n}\left(x_{0}\right)-f\left(x_{0}\right)\right)=\sqrt{\frac{k}{n}} \sqrt{n h^{p}}\left(\widehat{f}_{n}\left(x_{0}\right)-f\left(x_{0}\right)\right) .
$$

Under our assumptions on $K$ and $f$ one can easily verify that $\sqrt{n h^{p}}\left(\widehat{f}_{n}\left(x_{0}\right)-f\left(x_{0}\right)\right)=O_{\mathbb{P}}(1)$ (see e.g. Parzen, 1962) and hence the theorem follows.

\subsection{Proof of Theorem 2.2}

Let

$$
\mathcal{I}_{n}:=\left\{g_{\theta, \delta, n}: \theta \in \Theta, \delta \in H\right\}
$$

where for $\theta \in \Theta:=[0, T]^{d}$, and $\delta \in H:=\left\{\delta=\left(\delta_{1}, \ldots, \delta_{d}\right) ; \delta_{j}: \mathbb{R} \times S_{X} \rightarrow \mathbb{R}\right\}$ with

$$
\begin{aligned}
g_{\theta, \delta, n}(u, z) & :=\sqrt{\frac{n}{k} h^{p}} K_{h}\left(x_{0}-z\right) q_{\theta, \delta, n}(u, z) \\
& :=\sqrt{\frac{n}{k} h^{p}} K_{h}\left(x_{0}-z\right) \mathbb{1}_{\left\{1-\delta_{1}\left(u_{1}, z\right) \leqslant k / n \theta_{1} \text { or } \ldots \text { or } 1-\delta_{d}\left(u_{d}, z\right) \leqslant k / n \theta_{d}\right\}} .
\end{aligned}
$$

For convenience, denote $\delta_{n}:=\left(\widehat{F}_{n, 1}, \ldots, \widehat{F}_{n, d}\right)$ and $\delta_{0}:=\left(F_{1}, \ldots, F_{d}\right)$. According to Lemma 2.3, $r_{n}^{-1}\left|\delta_{n}-\delta_{0}\right|$ converges in probability towards the null function $H_{0}:=\{0\}$ in $H$ endowed with the norm $\|\delta\|_{H}:=\sum_{i=1}^{d}\left\|\delta_{i}\right\|_{\infty}$. In order to apply Theorem 2.3 in van der Vaart and Wellner (2007), we have now to show

Assertion 1: $\sup _{\theta \in \Theta} \sqrt{n} P G_{n}\left(\theta, a_{n}\right) \longrightarrow 0$ for every $a_{n} \rightarrow 0$, 
Assertion 2: $\sup _{\theta \in \Theta}\left|\mathbb{G}_{n} G_{n}(\theta, a)\right| \stackrel{\mathbb{P}}{\longrightarrow} 0$, for every $a>0$, where $G_{n}(\theta, a)$ is an envelope function for the class

$$
\mathcal{G}_{n}(\theta, a):=\left\{g_{\theta, \delta_{0}+r_{n} \delta, n}-g_{\theta, \delta_{0}, n}: \delta \in H,\|\delta\|_{H} \leqslant a\right\}
$$

Proof of Assertion 1. Using the ideas of the proof of Theorem 2.1, for any $\delta \in H$ such that $\|\delta\|_{H} \leqslant a$

$$
\begin{aligned}
\left|q_{\theta, \delta_{0}+r_{n} \delta, n}-q_{\theta, \delta_{0}, n}\right|(u, z) & \leqslant \mathbb{1}_{\left\{k / n \theta_{1}-r_{n} a \leqslant 1-F_{1}\left(u_{1} \mid z\right) \leqslant k / n \theta_{1}+r_{n} a \text { or } \ldots \text { or } k / n \theta_{d}-r_{n} a \leqslant 1-F_{d}\left(u_{d} \mid z\right) \leqslant k / n \theta_{d}+r_{n} a\right\}} \\
& =: \mathbb{1}_{B_{n, \theta, a}}(u, z) .
\end{aligned}
$$

Thus, we set $G_{n}(\theta, a)(u, z):=\sqrt{\frac{n}{k} h^{p}} K_{h}\left(x_{0}-z\right) \mathbb{1}_{B_{n, \theta, a}}(u, z)$ and we have

$$
\sqrt{n} P G_{n}\left(\theta, a_{n}\right)=n \sqrt{\frac{h^{p}}{k}} \int_{S_{K}} K(u) \mathbb{P}\left(B_{n, \theta, a_{n}} \mid X=x_{0}-h u\right) f\left(x_{0}-h u\right) d u,
$$

with

$$
\begin{aligned}
\mathbb{P}\left(B_{n, \theta, a_{n}} \mid X=x_{0}-h u\right) & \leqslant \sum_{j=1}^{d} \mathbb{P}\left(k / n \theta_{j}-r_{n} a_{n} \leqslant 1-F_{j}\left(Y^{(j)} \mid X\right) \leqslant k / n \theta_{j}+r_{n} a_{n} \mid X=x_{0}-h u\right) \\
& \leqslant 2 d r_{n} a_{n} .
\end{aligned}
$$

Hence,

$$
\sup _{\theta \in \Theta} \sqrt{n} P G_{n}\left(\theta, a_{n}\right) \leqslant 2 d n \sqrt{\frac{h^{p}}{k}} r_{n} a_{n}\left(f\left(x_{0}\right)+o(1)\right) \rightarrow 0
$$

and the assertion follows.

Proof of Assertion 2. The idea is to apply Lemma 2.2 in van der Vaart and Wellner (2007). Now we work with the class of functions $\left\{G_{n}(\theta, a), \theta \in[0, T]^{d}\right\}$, for any $a>0$ with the envelope function

$$
E_{n}(u, z):=\sqrt{\frac{n}{k} h^{p}} K_{h}\left(x_{0}-z\right) \mathbb{1}_{\left\{1-F_{1}\left(u_{1} \mid z\right) \leqslant k / n T+r_{n} a \text { or } \ldots \text { or } 1-F_{d}\left(u_{d} \mid z\right) \leqslant k / n T+r_{n} a\right\}} .
$$

Consequently, we have first to prove that

$$
\begin{array}{r}
\sup _{\theta \in \Theta} P G_{n}(\theta, a)^{2} \longrightarrow 0, \\
P E_{n}^{2}=O(1), \\
P E_{n}^{2} 1_{\left\{E_{n} \geqslant \epsilon \sqrt{n}\right\}} \rightarrow 0 \text { for every } \varepsilon>0 .
\end{array}
$$


For what concerns condition (16), we have according to (14)

$$
\begin{aligned}
P G_{n}(\theta, a)^{2} & =\int_{S_{K}} K(u)^{2} \frac{n}{k} \mathbb{P}\left(B_{n, \theta, a} \mid X=x_{0}-h u\right) f\left(x_{0}-h u\right) d u \\
& \leqslant 2 d \frac{n}{k} r_{n} a \int_{S_{K}} K(u)^{2} f\left(x_{0}-h u\right) d u \\
& =2 d \frac{n}{k} r_{n} a\left(f\left(x_{0}\right)\|K\|_{2}^{2}+o(1)\right) .
\end{aligned}
$$

We have that $r_{n} n / k \rightarrow 0$ since $n \sqrt{h^{p} / k} r_{n}$ converges and $k h^{p} \rightarrow \infty$, and as such (16) is established.

By the first order condition, we have

$$
\begin{aligned}
P E_{n}^{2} & =\int_{S_{K}} K(u)^{2} \frac{n}{k} \mathbb{P}\left(A_{n / k, y_{T}+(n / k) r_{n} y_{a}} \mid X=x_{0}-h u\right) f\left(x_{0}-h u\right) d u \\
& =L\left(y_{T} \mid x_{0}\right) f\left(x_{0}\right)\|K\|_{2}^{2}+o(1),
\end{aligned}
$$

where $y_{a}:=(a, \ldots, a) \in \mathbb{R}_{+}^{d}$ and (17) follows.

Now we verify condition (18). For any $\xi>0$ we obtain the following inequality

$$
\begin{aligned}
P E_{n}^{2} \mathbb{1}_{\left\{E_{n} \geqslant \epsilon \sqrt{n}\right\}} & \leqslant \frac{1}{\varepsilon^{\xi} n^{\xi / 2}} P E_{n}^{2+\xi} \\
& \leqslant \frac{1}{\varepsilon^{\xi}} \frac{d}{\left(k h^{p}\right)^{\xi / 2}}\left(T+r_{n} \frac{n}{k} a\right)\left(\|K\|_{2+\xi}^{2+\xi} f\left(x_{0}\right)+o(1)\right),
\end{aligned}
$$

which tends to zero under the assumptions of the theorem.

It remains to show that

$$
J\left(d_{n},\left\{G_{n}(\theta, a): \theta \in \Theta\right\}, L_{2}\right) \longrightarrow 0 \text { for all } d_{n} \searrow 0
$$

To deal with the uniform entropy integral, we can reuse the lines of proof of Theorem 2.1 by considering the following class of functions on $[0,1]^{d}$

$$
\left.\left\{u \rightarrow \mathbb{1}_{\left\{y_{1} \leqslant 1-u_{1} \leqslant y_{2}\right.} \text { or } \ldots \text { or } y_{2 d-1} \leqslant 1-u_{d} \leqslant y_{2 d}\right\}, \quad y_{1}<y_{2}, \ldots, y_{2 d-1}<y_{2 d}\right\},
$$

which is a $V C$-class since the class of sets associated to the indicator functions is a $V C$-class of sets as the union of $d V C$-classes of sets. This allows us to prove that there exist positive constants $C$ and $V$ such that

$$
\sup _{Q \in \mathcal{Q}} N\left(\left\{G_{n}(\theta, a): \theta \in \Theta\right\}, L_{2}(Q), \tau\left\|E_{n}\right\|_{Q, 2}\right) \leqslant C\left(\frac{1}{\tau}\right)^{V},
$$

from which the last assertion follows. This achieves the proof of Theorem 2.2. 


\subsection{Proof of Theorem 2.3}

Due to the decomposition (4), we have to prove that

$$
\sup _{y \in[0, T]^{d}} \sqrt{k h^{p}}\left|\mathbb{E}\left[\breve{T}_{k}\left(y \mid x_{0}\right)-\widehat{T}_{k}\left(y \mid x_{0}\right)\right]\right|=o(1) .
$$

According to the notation in the proof of Theorem 2.2, note that $\sqrt{k h^{p}} \mathbb{E}\left[\left|\breve{T}_{k}-\widehat{T}_{k}\right|\right]\left(y \mid x_{0}\right)$ equals

$$
\begin{aligned}
& \sqrt{n} \mathbb{E}\left[\mid \frac{1}{n} \sum_{i=1}^{n}\left[\sqrt{\frac{n}{k} h^{p}} K_{h}\left(x_{0}-X_{i}\right) \mathbb{1}_{\left\{1-\widehat{F}_{n, 1}\left(Y_{i}^{(1)} \mid X_{i}\right) \leqslant k / n y_{1} \text { or } \ldots \text { or } 1-\hat{F}_{n, d}\left(Y_{i}^{(d)} \mid X_{i}\right) \leqslant k / n y_{d}\right\}}\right.\right. \\
- & \sqrt{\frac{n}{k} h^{p}} K_{h}\left(x_{0}-X_{i}\right) \mathbb{1}_{\left.\left.\left.\left\{1-F_{1}\left(Y_{i}^{(1)} \mid X_{i}\right) \leqslant k / n y_{1} \text { or } \ldots \text { or } 1-F_{d}\left(Y_{i}^{(d)} \mid X_{i}\right) \leqslant k / n y_{d}\right\}\right]\right]\right]} \\
\leqslant & \sqrt{n} \mathbb{E}\left[\left|g_{y, \delta_{n}, n}\left(Y_{1}, X_{1}\right)-g_{y, \delta_{0}, n}\left(Y_{1}, X_{1}\right)\right|\right] \\
\leqslant & \sqrt{n} P G_{n}(y, a),
\end{aligned}
$$

for $n$ large enough, since with probability tending to $1, \delta_{n} \in \delta_{0}+r_{n} \mathcal{B}(0, a)$ where $\mathcal{B}(0, a):=\{\delta$ : $\left.\|\delta\|_{H} \leqslant a\right\}$, and by using the Skorohod representation. This implies that

$$
\sup _{y \in[0, T]^{d}} \sqrt{k h^{p}} \mathbb{E}\left[\left|\breve{T}_{k}-\widehat{T}_{k}\right|\right]\left(y \mid x_{0}\right) \leqslant \sup _{y \in[0, T]^{d}} \sqrt{n} P G_{n}(y, a) \rightarrow 0,
$$

by Assertion 1 since it is clear that $a_{n} \rightarrow 0$ can be replaced by any fixed value $a$ in (15) and conclude with the fact that $n r_{n} \sqrt{h^{p} / k} \rightarrow 0$.

Finally,

$$
\left\{\sqrt{k h^{p}}\left(\frac{\breve{T}_{k}\left(y \mid x_{0}\right)}{\widehat{f}_{n}\left(x_{0}\right)}-L\left(y \mid x_{0}\right)-\alpha_{x_{0}}\left(\frac{n}{k}\right) M_{x_{0}}(y)\right), y \in[0, T]^{d}\right\},
$$

can be handled using the same arguments as those at the end of the proof of Theorem 2.1.

\section{Acknowledgement}

This work was supported by a research grant (VKR023480) from VILLUM FONDEN and an international project for scientific cooperation (PICS-6416). The authors sincerely thank the editor, associate editor and the referees for their helpful comments and suggestions that led to substantial improvement of the paper. 


\section{References}

[1] Beirlant, J., Dierckx, G., Guillou, A., 2011. Bias-reduced estimators for bivariate tail modelling. Insurance: Mathematics and Economics, 49, 18-26.

[2] Beirlant, J., Escobar-Bach, M., Goegebeur, Y., Guillou, A., 2016. Bias-corrected estimation of stable tail dependence function. Journal of Multivariate Analysis, 143, 453-466.

[3] Beirlant, J., Vandewalle, B., 2002. Some comments on the estimation of a dependence index in bivariate extreme value in statistics. Statistics and Probability Letters, 60, 265-278.

[4] Bücher, A., Segers, J., Volgushev, S., 2014. When uniform weak convergence fails: empirical processes for dependence functions and residuals via epi- and hypographs. Annals of Statistics, 42, 1598-1634.

[5] Capéraà, P., Fougères, A. L., Genest, C., 2000. Bivariate distributions with given extreme value attractor. Journal of Multivariate Analysis, 72, 30-49.

[6] de Carvalho, M., Davison, A.C., 2014. Spectral density ratio models for multivariate extremes. Journal of the American Statistical Association, 109, 764-776.

[7] Coles, S.G., Heffernan, J.E., Tawn, J.A., 1999. Dependence measures for extreme value analyses. Extremes, 2, 339-365.

[8] Daouia, A., Gardes, L., Girard, S., 2013. On kernel smoothing for extremal quantile regression. Bernoulli, 19, 2557-2589.

[9] Daouia, A., Gardes, L., Girard, S., Lekina, A., 2011. Kernel estimators of extreme level curves. Test, 20, 311-333.

[10] Dekkers, A.L.M., Einmahl, J.H.J., de Haan, L., 1989. A moment estimator for the index of an extreme-value distribution. Annals of Statistics, 17, 1833-1855.

[11] Drees, H., Huang, X., 1998. Best attainable rates of convergence for estimators of the stable tail dependence function. Journal of Multivariate Analysis, 64, 25-47. 
[12] Dutang, C., Goegebeur, Y., Guillou, A., 2014. Robust and bias-corrected estimation of the coefficient of tail dependence. Insurance: Mathematics and Economics, 57, 46-57.

[13] Einmahl, J.H.J., de Haan, L., Sinha, A.K., 1997. Estimating the spectral measure of an extreme value distribution. Stochastic Processes and their Applications, 70, 143-171.

[14] Escobar-Bach, M., Goegebeur, Y., Guillou, A., 2017a. Local robust estimation of the Pickands dependence function. https://hal.archives-ouvertes.fr/hal-01340166.

[15] Escobar-Bach, M., Goegebeur, Y., Guillou, A., You, A., 2017b. Bias-corrected and robust estimation of the bivariate stable tail dependence function. Test, 26, 284-307.

[16] Fils-Villetard, A., Guillou, A., Segers, J., 2008. Projection estimators of Pickands dependence functions. Canadian Journal of Statistics, 36, 369-382.

[17] Fougères, A.L., de Haan, L., Mercadier, C., 2015. Bias correction in multivariate extremes. Annals of Statistics, 43, 903-934.

[18] Gannoun, A., Girard, S., Guinot, C., Saracco, J., 2002. Reference ranges based on nonparametric quantile regression. Statistics in Medicine, 21, 3119-3135.

[19] Giné, E., Guillou, A., 2002. Rates of strong uniform consistency for multivariate kernel density estimators. Annales de l'Institut Henri Poincaré - Probabilités et Statistiques, 38, 907-921.

[20] Goegebeur, Y., Guillou, A., 2013. Asymptotically unbiased estimation of the coefficient of tail dependence. Scandinavian Journal of Statistics, 40, 174-189.

[21] Goegebeur, Y., Guillou, A., Osmann, M., 2014a. A local moment type estimator for the extreme value index in regression with random covariates. Canadian Journal of Statistics, $42,487-507$.

[22] Goegebeur, Y., Guillou, A., Rietsch, T., 2015. Robust conditional Weibull-type estimation. Annals of the Institute of Statistical Mathematics, 67, 479-514. 
[23] Goegebeur, Y., Guillou, A., Schorgen, A., 2014b. Nonparametric regression estimation of conditional tails - the random covariate case. Statistics, 48, 732-755.

[24] de Haan, L., Ferreira, A., 2006. Extreme Value Theory - An Introduction, Springer.

[25] Hall, P., Tajvidi, N., 2000. Distribution and dependence-function estimation for bivariate extreme-value distributions. Bernoulli, 6, 835-844.

[26] Hill, B.M., 1975. A simple general approach to inference about the tail of a distribution. Annals of Statistics, 3, 1163-1174.

[27] Huang, X., 1992. Statistics of bivariate extremes. PhD Thesis, Erasmus University Rotterdam, Tinbergen Institute Research series No. 22.

[28] Ledford, A.W., Tawn, J.A., 1997. Modelling dependence within joint tail regions. Journal of the Royal Statistical Society Series B, 59, 475-499.

[29] Mahmud, A., Tyree, M., Cayan, D., Motallebi, N., Kleeman, M.J., 2008. Statistical downscaling of climate change impacts on ozone concentrations in California. Journal of Geophysical Research, 113, doi:10.1029/2007JD009534.

[30] Parzen, E., 1962. On estimation of a probability density function and mode. Annals of Mathematical Statistics, 33, 1065-1076.

[31] Peng, L., 1999. Estimation of the coefficient of tail dependence in bivariate extremes. Statistics and Probability Letters, 43, 399-409.

[32] Peng, L., 2010. A practical way for estimating tail dependence functions. Statistica Sinica, $20,365-378$.

[33] Pickands, J., 1975. Statistical inference using extreme order statistics. Annals of Statistics, $3,119-131$.

[34] Pickands, J., 1981. Multivariate extreme value distributions. Bulletin of the International Statistical Institute, 49, 859-878. 
[35] Portier, F., Segers, J., 2015. On the weak convergence of the empirical conditional copula under a simplifying assumption. ArXiv:1511.06544.

[36] Steiner, A.L., Davis, A.J., Sillman, S., Owen, R.C., Michalak, A.M., Fiore, A.M., 2010. Observed suppression of ozone formation at extremely high temperatures due to chemical and biophysical feedbacks. Proceedings of the National Academy of Sciences, 107, 1968519690.

[37] Stupfler, G., 2013. A moment estimator for the conditional extreme-value index. Electronic Journal of Statistics, 7, 2298-2353.

[38] van der Vaart, A.W., 1998. Asymptotic statistics. Cambridge Series in Statistical and Probabilistic Mathematics, Cambridge University Press, Cambridge.

[39] van der Vaart, A.W., Wellner, J.A., 1996. Weak convergence and empirical processes, with applications to statistics. Springer Series in Statistics, Springer-Verlag, New York.

[40] van der Vaart, A.W., Wellner, J.A., 2007. Empirical processes indexed by estimated functions. Asymptotics: Particles, Processes and Inverse Problems, IMS Lecture Notes Monograph Series, 55, 234-252.

[41] Yao, Q., 1999. Conditional predictive regions for stochastic processes. Technical report, University of Kent at Canterbury. 